\title{
Impact of Segmentation and Capture on Slotted Aloha Systems Exploiting Interference Cancellation
}

\author{
F. Babich, Senior Member, IEEE, and M. Comisso, Member, IEEE \\ Department of Engineering and Architecture, University of Trieste, Via A. Valerio 10, 34127 Trieste, Italy \\ E-mail: babich (mcomisso) dunits.it
}

\begin{abstract}
The performance of the widely adopted slotted Aloha (SA) scheme has been recently improved thanks to the introduction of novel mechanisms, including interference cancellation (IC), packet segmentation, and slot slicing. The combined effect of these mechanisms in the presence of capture has however not yet deeply investigated, even if the resulting impact on the network behavior is determinant for properly quantifying the achievable throughput. To deal with this issue, this paper analyzes the influence of capture on a framed SA (FSA) system adopting IC, segmentation, and slicing, by considering a reliable decoding criterion that accounts for the actually experienced signal to interference-plus-noise ratio. A theoretical model is developed to evaluate the capture probability in fast and slow Rayleigh fading conditions, deriving closed-form expressions for the interferencelimited case. The IC-based FSA throughput is then estimated adopting a Markov chain approach validated by Monte Carlo simulations. Finally, the performance of an actual system using a quadrature phase-shift keying modulation in conjunction with a turbo encoder is compared with that estimated by adopting the considered decoding criterion, in order to check its applicability to practical communication networks.
\end{abstract}

Index Terms-Framed slotted Aloha, capture, packet segmentation, slot slicing, interference cancellation.

\section{INTRODUCTION}

Since several years, the need of managing network scenarios where the nodes cannot be coordinated by a centralized authority has led to the adoption of random access protocols, with the aim of enabling the network to operate in the presence of irregular and/or inhomogeneous traffic conditions [1]. Differently from traditional frequency, time, or code division multiple access mechanisms, which may provide a larger performance but require a coordination among the nodes, random access schemes allow the sharing of the channel resources by a population of users when a sufficient level of coordination cannot be achieved [2]. Scenarios characterized by this limitation may derive from the sporadic activity of the nodes or from their excessive number, as well as from the unavailability of global information or the too large communication delays. The development of random access solutions has hence become a relevant topic in wired and wireless communication research involving satellite, cellular, local and personal area networks.

\section{A. Related work}

In the random access context, even if several solutions have been conceived along the years [1-3], two main families of

\footnotetext{
${ }^{1}$ This work is partly supported by the Italian Ministry of University and Research (MIUR), project FRA 2018 (Univ. of Trieste): "UBER-5G: Cubesat $5 \mathrm{G}$ networks - Access layer analysis and antenna system development".
}

algorithms are at present physically implemented on network devices: one relying on the slotted Aloha (SA) scheme, and another one relying on the carrier sensing multiple access (CSMA) one. To achieve a higher throughput with respect to SA, CSMA requires a propagation environment in which the carrier signal can be reliably detected, so as to provide an updated information to the access layer [4]. Unfortunately, this requirement cannot be usually satisfied in satellite and cellular scenarios, where SA remains the preferable solution despite its efficiency lies well below the unity [5].

A slight SA improvement, called diversity SA (DSA), has been proposed in [6], by introducing diversity through the transmission of multiple copies of the same packet. However, only in the last years a major breakthrough has been made with the implementation of interference cancellation (IC) techniques [7-9]. These techniques enable to constructively exploit the collisions by canceling the interference due to the correctly received packets, in order to allow the possible recovery of other initially collided packets. IC has immediately gained interest in the cellular context for separating multiple superimposed signals, with the aim of enabling non-orthogonal multiple access (NOMA) [10], and non-orthogonal random access (NORA) mechanisms [11]. A first algorithm combining DSA and IC is the contention resolution DSA (CRDSA) [7]. In this scheme, two copies of each packet are transmitted to increase the success probability for small loads, and IC is used to cancel from the collided slots the interference caused by the packets correctly detected in other slots. The IC process becomes hence iterative, with the iteration one that corresponds to DSA. The step ahead CRDSA is represented by the irregular repetition SA (IRSA) [8], in which the number of transmitted packet copies is not fixed, but is selected according to an optimized probability mass function (pmf). The subsequent generalization of IRSA is the coded SA (CSA) [9], in which a packet is encoded, rather than simply repeated. More precisely, the packet is split into $H$ data segments that are encoded to produce $L$ segments of identical length, where $L$ is selected according to a given pmf. Besides, each slot is subdivided into $L$ slices, each having the same duration of the transmission time of a segment. Thus, in the CSA scheme, the minimum resource unit that can be allocated for a transmission is no longer the slot, but the slice, and a packet transmission is considered successful whether at least $H$ segments are correctly received.

This evolution reveals that SA has been gradually improved by adding ever novel features, from packet repetition and 
IC, to slot slicing, packet coding and segmentation. Within this context, the usual approach for modeling the result of a transmission relies on considering as successful a slot/slice with a unique packet/segment that provides the entire information it contains. Conversely, a collided slot/slice is assumed to provide no information. In a real scenario, both these assumptions may not hold, since the propagation channel effects may damage an uncollided packet/segment or, through capture, may allow the reception of one or more collided ones. This has led to the development of a significant parallel branch of research, not strictly focused on the mechanisms for improving SA, but aimed at reliably modeling the SA throughput initially considering the sole path-loss attenuation [5], and then including Rayleigh [12], [13], Rice [14], or Nakagami fading [15], and also log-normal shadowing [16]. These studies provide mathematical foundations to the possibility of increasing the SA performance by exploiting the capture effect that may spontaneously occur in a real radio receiving system.

\section{B. Motivation and contribution}

With specific reference to the novel IC-based SA schemes, the capture effect has been outlined for the sole IRSA case [8], while the study of the SA performance in the presence capture, IC, segmentation, and slicing still represents an open issue. This latter task requires to adopt a decoding criterion not simply based on the signal to interference-plus-noise ratio (SINR) of a single segment, but capable to jointly account for the SINRs of all the segments belonging to a packet. Even a collided segment, in fact, might provide a residual information, which can be associated with that provided by other segments of the same packet to possibly recover the packet itself. Therefore, the capture of a packet becomes no more simply determined by the clean reception of at least $H$ over $L$ segments, as in the common erasure channel model, but by the joint, manifold amount of information provided by all the $L$ segments, collided and uncollided.

A proper modeling of this situation is the objective of this paper, which analyzes the combined effect of capture, segmentation, slicing, and IC on the throughput of a framed SA (FSA) scheme, in order to provide some useful insights concerning the expected upgrade of the current FSA implementations to the advanced IC-based functionalities [17]. To this aim, three contributions are provided. Firstly, moving from the concept of equivalent rate [18], which models the actual rate of a single communication, a novel decoding criterion, suitable to model the capture channel in the presence of segmentation, is formulated. Secondly, based on this criterion, a theoretical analysis is developed to derive the capture probability in both fast and slow Rayleigh fading scenarios. This analysis provides closed-form expressions for the interference-limited case, which typically occurs in cellular and broadcasting radio systems. A two-dimensional Markov model, which is validated by exhaustive Monte Carlo simulations, is also conceived to evaluate, for each iteration, the throughput of an FSA system adopting IC. As a third contribution, the practical usefulness of the formulated decoding criterion is investigated by considering the performance of an actual system using a quadrature phase-shift keying (QPSK) modulation combined with a turbo encoder. Finally, some implementation issues concerning the required receiver operations and the information necessary to make the segmentation process feasible are discussed. To the best of authors' knowledge, the here proposed analysis represents, in the context of uncoordinated random access, the first attempt of modeling the benefits of segmentation on an IC-enabled FSA system with capture by allowing the throughput estimation at each IC iteration.

The paper is organized as follows. The system model is introduced in Section II. The equivalent rate statistic is derived in Section III. The capture and throughput analyses are developed in Section IV. The numerical results are presented in Section V. The implementation issues are discussed in Section VI. The main conclusions are summarized in Section VII.

\section{SYSTEM MODEL}

Consider $N$ contending users that have to send their packets to a common destination using an FSA random access scheme. In agreement with the framed assumption, the time is subdivided in random access frames (RAFs) of $K$ slots, and each user, assumed frame- and slot-synchronous, attempts the transmission of at most one packet in each RAF. The generic slot is further subdivided into $L$ slices of identical duration, while each packet is first encoded through a channel encoder of rate $\rho(0<\rho \leq 1)$ and then split into $L$ segments of identical size. Assuming the guard times as negligible [9], the transmission time of the encoded packet (set of the whole $L$ segments) is set equal to the slot duration, while the transmission time of the single segment is set equal to the slice duration. The adopted code is assumed symmetric, in the sense that the original information provided by the uncoded packet is uniformly spread across the $L$ segments, so as each encoded segment gives the same contribution to the decoding process. This symmetry requirement may be reasonably well satisfied by properly designed turbo codes [19]. Observe also that these encoding rules, which are adopted to specifically analyze the impact of segmentation, partly differ from the CSA ones. In fact, in [9], the generic uncoded packet is first split into $H$ information segments of identical size, which are subsequently encoded to obtain $L(\geq H)$ segments, all of the same size as the information segments, but where $L$ is randomly selected at each transmission attempt according to a given pmf. Thus, in [9], different packets are encoded with different code rates, while, in the here investigated scenario, all users operate using the same code rate $\rho$. This implies that, in the considered case, the code rate of the access scheme and the code rate of the packet coincide and are both equal to $\rho$. This choice is made to focus the analysis on packet segmentation, while maintaining its mathematical tractability in the presence of fading. In terms of practical application, the use of a unique code rate is even simpler to implement with respect to code diversity. In fact, in real systems, multiple rates are usually obtained by adopting a unique encoder operating at the minimum rate and some perforation matrices removing the redundancy necessary to achieve a desired higher rate. In the case of single code rate, the perforation is not necessary. The benefits provided by code 
diversity are however discussed in Section VI by numerically estimating the impact of the different kinds of diversity on the throughput. Besides, an analysis of the capture probability for the CSA scheme, including both segmentation and code diversity, has been recently presented in [20], but limiting the theoretical model to a constant power scenario.

Concerning the access rules, at each attempt the generic user randomly selects, among the $K \cdot L$ slices of the RAF, the $L$ ones in which its $L$ segments are transmitted. At the destination, when IC is employed, the segments, possibly collided but belonging to successfully decoded packets, are cancelled from the corresponding slices, thus enabling further packet decodings. To simplify the treatise of the theoretical analysis, the destination is ideally assumed to know, for each packet, the slices of the RAF in which the segments of that packet have been placed. This assumption will be removed in Section VI by discussing the impact on the performance due to the insertion of slice pointers in the segment header [9]. Regarding the cancellation process, this paper assumes an ideal channel estimation and hence a perfect IC. This is consistent with the results in [7] and [9], which prove that a nonideal channel estimation for IC purposes leads just to a slight performance degradation with respect to the ideal case. This aspect will be however further considered in Section VI by accounting for imperfect IC in the absence and in the presence of fading. Besides, according to [7-9], the entire decoding/IC process is assumed to be iterated until a specified maximum number of iterations, where the iteration one corresponds to the absence of IC and hence to the usage of the common FSA scheme in the presence of segmentation and capture.

Within the above described procedure, the usually adopted decoding model based on the packet erasure channel assumes that the destination decodes the packet if at least $H$ over $L$ of its segments are uncollided [9], that is, they lie in slices containing a unique segment. This approach has the limit of using a not realistic clear-cut binary decision for each segment, which does not allow one to account for the capture effect that jointly involves the $L$ segments. To overcome this limit, and hence enable a more detailed analysis of segmentation and slicing in FSA systems, a more advanced decoding criterion is formulated in the next subsection.

\section{A. Selected and equivalent rates}

The considered decoding criterion relies on the comparison between two quantities: the minimum rate required for the successful reception of the packet, and the average one experienced at the destination by considering all packet segments. The first quantity can be immediately defined for a bidimensional linear modulation of order $\xi$ and for the code rate $\rho$, as the number of information bits transmitted per symbol:

$$
\varrho=\rho \log _{2} \xi,
$$

which identifies the rate selected by each user for its transmission. The second quantity, called equivalent rate, involves the channel statistics and is defined as follows.

Consider the $l$-th segment $(l=1, \ldots, L)$ transmitted by a target user in a slice where a collision of order $M_{l}(0 \leq$
$M_{l} \leq N-1$ ) occurs. This implies that, together with the target user, other $M_{l}$ interfering users have transmitted a segment in the same slice, leading to $M_{l}+1$ colliding segments. With reference to this slice, denote as $S_{0}$ the random variable (r.v.) identifying the power received by the destination from the target user, as $S_{m}$ that representing the power received from the $m$-th interferer $\left(m=1, \ldots, M_{l}\right)$, and as $\varsigma$ the noise power. Besides, assume that $S_{0}, \ldots, S_{M_{l}}$ are independent and identically distributed (i.i.d.) exponential r.v.s characterized, for $m=0, \ldots, M_{l}$, by the probability density function (pdf):

$$
f_{S_{m}}\left(s_{m}\right)=\frac{1}{\zeta} \exp \left(-\frac{s_{m}}{\zeta}\right) \mathbb{1}_{\mathbb{R}_{0}^{+}}\left(s_{m}\right),
$$

where $\zeta$ is the average power, $\mathbb{R}_{0}^{+}$is the set of nonnegative real numbers, and $\mathbb{1}_{\mathbf{Y}}(\mathbf{y})$ is the indicator function, that is, $\mathbb{1}_{\mathbf{Y}}(\mathbf{y})=1$ if $\mathbf{y} \in \mathbf{Y}$ and $\mathbb{1}_{\mathbf{Y}}(\mathbf{y})=0$ if $\mathbf{y} \notin \mathbf{Y}$. According to this notation, the r.v. denoting the SINR of the $l$-th segment transmitted by the target user to the destination is given by:

$$
X_{l}=\frac{S_{0}}{U_{l}}
$$

where $U_{l}=\sum_{m=1}^{M_{l}} S_{m}+\varsigma$ denotes the interference-plus-noise power. This r.v. follows a translated Erlang distribution [21], whose cumulative distribution function (cdf) is:

$$
F_{U_{l}}\left(u_{l} ; M_{l}, \varsigma\right)=\frac{1}{\left(M_{l}-1\right) !} \gamma\left(M_{l}, \frac{u_{l}-\varsigma}{\zeta}\right) \mathbb{1}_{[\varsigma,+\infty)}\left(u_{l}\right),
$$

where $\gamma(.,$.$) is the lower incomplete gamma function [22].$ Observe that (4) holds also for $M_{l}=0$ (noise-limited case), since, for $M_{l} \rightarrow 0, F_{U_{l}}\left(u_{l} ; M_{l}, \varsigma\right) \rightarrow \mathbb{1}_{[\varsigma,+\infty)}\left(u_{l}\right)$ (degenerate distribution). Hence, using (3) and recalling the rule for the ratio between r.v.s, the cdf of $X_{l}$ is evaluated as [23]:

$$
\begin{aligned}
F_{X_{l}}\left(x_{l} ; M_{l}, \varsigma\right) & =\operatorname{Pr}\left\{X_{l} \leq x_{l}\right\}=1-\operatorname{Pr}\left\{U_{l}<s_{0} / x_{l}\right\} \\
& =1-\int_{0}^{+\infty} f_{S_{0}}\left(s_{0}\right) F_{U_{l}}\left(\frac{s_{0}}{x_{l}} ; M_{l}, \varsigma\right) \mathrm{d} s_{0} \\
& =\left[1-\exp \left(-\frac{\varsigma x_{l}}{\zeta}\right) \frac{1}{\left(x_{l}+1\right)^{M_{l}}}\right] \mathbb{1}_{\mathbb{R}_{0}^{+}}\left(x_{l}\right),
\end{aligned}
$$

from which one obtains the corresponding pdf as:

$$
\begin{aligned}
f_{X_{l}}\left(x_{l} ; M_{l}, \varsigma\right)=\frac{\mathrm{d} F_{X_{l}}}{\mathrm{~d} x_{l}}= & \exp \left(-\frac{\varsigma x_{l}}{\zeta}\right) \frac{1}{\left(x_{l}+1\right)^{M_{l}+1}} \\
& \cdot\left[\frac{\varsigma\left(x_{l}+1\right)}{\zeta}+M_{l}\right] \mathbb{1}_{\mathbb{R}_{0}^{+}}\left(x_{l}\right) .
\end{aligned}
$$

The rate achievable for a SINR $X_{l}$ when a bidimensional linear modulation is adopted may be then established by considering the Shannon bound. Accordingly, the r.v. representing the rate for the $l$-th segment is related to $X_{l}$ by:

$$
\Lambda_{l}=\log _{2}\left(1+X_{l}\right)
$$

Since this latter expression is invertible, one directly obtains:

$$
F_{\Lambda_{l}}\left(\lambda_{l} ; M_{l}, \varsigma\right)=\operatorname{Pr}\left\{\Lambda_{l} \leq \lambda_{l}\right\}=F_{X_{l}}\left(2^{\lambda_{l}}-1 ; M_{l}, \varsigma\right),
$$

which, by (5) and (6), provides the cdf and pdf of $\Lambda_{l}$ as:

$$
F_{\Lambda_{l}}\left(\lambda_{l} ; M_{l}, \varsigma\right)=\left\{1-\exp \left[-\frac{\varsigma\left(2^{\lambda_{l}}-1\right)}{\zeta}\right] 2^{-M_{l} \lambda_{l}}\right\} \mathbb{1}_{\mathbb{R}_{0}^{+}}\left(\lambda_{l}\right),
$$




$$
F_{R}\left(r ; \mathbf{M}_{L}, \varsigma\right)=\int_{-\infty}^{+\infty} \ldots \int_{-\infty}^{+\infty} F_{\Lambda_{L}}\left(L r-\sum_{l=1}^{L-1} \lambda_{l} ; M_{L}, \varsigma\right) \prod_{l=1}^{L-1} f_{\Lambda_{l}}\left(\lambda_{l} ; M_{l}, \varsigma\right) \mathrm{d} \lambda_{1} \ldots \mathrm{d} \lambda_{L-1}
$$

$$
\begin{aligned}
f_{\Lambda_{l}}\left(\lambda_{l} ; M_{l}, \varsigma\right)= & \log 2 \exp \left[-\frac{\varsigma\left(2^{\lambda_{l}}-1\right)}{\zeta}\right] 2^{-M_{l} \lambda_{l}} \\
& \cdot\left(\frac{\varsigma 2^{\lambda_{l}}}{\zeta}+M_{l}\right) \mathbb{1}_{\mathbb{R}_{0}^{+}}\left(\lambda_{l}\right) .
\end{aligned}
$$

Once the rate statistics of the $L$ segments of a packet are available, one can evaluate the cdf of their mean in order to obtain a rate comparable to the selected one. This allows the formulation of the following decoding criterion.

Packet capture criterion. A packet is successfully decoded if its equivalent rate [24]:

$$
R=\frac{1}{L} \sum_{l=1}^{L} \Lambda_{l}
$$

is larger or equal to the rate $\varrho$ in (1) selected by the user for its transmission.

The objective of this criterion is to overcome the limitations of the erasure channel for modeling the capture effect in a contention-based scenario using segmentation and IC. Note that (11) is not defined for estimating the information-theoretic achievable rate [25], since the derivation of possible capacity bounds is out of the scope of this paper, but to model the actual behavior of an efficient code (i.e., turbo or low density parity check), during packet transmission in the presence of interference and consistently with the propagation environment [24]. A detailed check of the accuracy of this criterion in reliably estimating the result of a transmission will be carried out in Subsection V-C by adopting a QPSK modulation and a turbo encoder operating on real sequences of bits. In this regard, observe that the result of the reception depends on the vector $\mathbf{M}_{L}=\left[M_{1}, \ldots, M_{L}\right]$, whose components are the collision orders experienced by the $L$ segments of the packet in the respective slices. From now on, this vector will be referred to as the interference context of the packet. According to this definition, the capture probability of a packet transmitted at a selected rate $\varrho$ using $L$ encoded segments in an interference context $\mathbf{M}_{L}$ is obtained as:

$$
c\left(\varrho ; \mathbf{M}_{L}, \varsigma\right)=\operatorname{Pr}\{R \geq \varrho\}=1-F_{R}\left(\varrho ; \mathbf{M}_{L}, \varsigma\right),
$$

where $F_{R}\left(r ; \mathbf{M}_{L}, \varsigma\right)$ denotes the cdf of $R$.

\section{EQUIVALENT RATE ANALYSIS}

To provide a deeper view of the meaning of (11) and (12), two relevant scenarios are analyzed. The first one is a fast fading scenario, where, in a RAF, $S_{0}$ may be different for each segment of a packet, that is, in the $L$ slices, the $L$ segments of a packet can be received with $L$ different powers corresponding to $L$ independent realizations of $S_{0}$. The second one is a slow fading scenario, where the realization of $S_{0}$ remains constant in the RAF, that is, in the $L$ slices, the $L$ segments of a packet are received with the same power corresponding to a unique realization of $S_{0}$. Therefore, since the power of a segment is assumed constant within a slice, the terms 'slow' and 'fast' are referred to the RAF duration, in which the channel varies among different slices in the fast fading case and remains instead constant in the slow fading one. Since, by (3) and (7), the generic rate $\Lambda_{l}$ depends on $S_{0}, L$ independent realizations of $S_{0}$ leads to $L$ independent rates $\Lambda_{1}, \ldots, \Lambda_{L}$ when fading is fast, while a unique realization of $S_{0}$ introduces correlation among these $L$ rates when fading is slow. The following subsections investigate in detail these two situations by deriving analytical expressions for the corresponding equivalent rate statistics.

\section{A. Fast fading scenario}

In the fast fading scenario, the $L$ r.v.s $\Lambda_{1}, \ldots, \Lambda_{L}$ are independent, thus their conditional statistics coincide with the unconditional ones. By consequence, the cdf of $R$ in (11), whose evaluation requires $L-1$ convolutions and a scaling, is obtained from (13) by using $F_{\Lambda_{l}}\left(\lambda_{l} ; M_{l}, \varsigma\right)$ in (9) and $f_{\Lambda_{l}}\left(\lambda_{l} ; M_{l}, \varsigma\right)$ in (10).

In general, (13) must be numerically evaluated. However, interestingly, some closed-form expressions may be found when $\varsigma=0$ (interference-limited case). In the absence of noise, in fact, (9) and (10) can be rewritten, respectively, as:

$$
\begin{gathered}
F_{\Lambda_{l}}\left(\lambda_{l} ; M_{l}, 0\right)=\left(1-2^{-M_{l} \lambda_{l}}\right) \mathbb{1}_{\mathbb{R}_{0}^{+}}\left(\lambda_{l}\right), \\
f_{\Lambda_{l}}\left(\lambda_{l} ; M_{l}, 0\right)=\log 2 M_{l} 2^{-M_{l} \lambda_{l}} \mathbb{1}_{\mathbb{R}_{0}^{+}}\left(\lambda_{l}\right),
\end{gathered}
$$

which allow one to solve (13) for any $L$ value. To this aim, consider first $L=1$, which immediately leads to $F_{R}\left(r ; \mathbf{M}_{1}, 0\right)=F_{\Lambda_{1}}\left(r ; M_{1}, 0\right), f_{R}\left(r ; \mathbf{M}_{1}, 0\right)=f_{\Lambda_{1}}\left(r ; M_{1}, 0\right)$. For $L=2$, (13) is rewritten using (14) and (15) as:

$$
\begin{aligned}
F_{R}\left(r ; \mathbf{M}_{2}, 0\right) & =\log 2 M_{1} \int_{0}^{2 r}\left[1-2^{-M_{2}\left(2 r-\lambda_{1}\right)}\right] 2^{-M_{1} \lambda_{1}} \mathrm{~d} \lambda_{1} \\
& =\left(1-\frac{M_{2} \cdot 2^{-2 M_{1} r}}{M_{2}-M_{1}}-\frac{M_{1} \cdot 2^{-2 M_{2} r}}{M_{1}-M_{2}}\right) \mathbb{1}_{\mathbb{R}_{0}^{+}}(r),
\end{aligned}
$$

whose derivative provides the corresponding pdf:

$$
f_{R}\left(r ; \mathbf{M}_{2}, 0\right)=\frac{2 \log 2 M_{1} M_{2}}{M_{2}-M_{1}}\left(2^{-2 M_{1} r}-2^{-2 M_{2} r}\right) \mathbb{1}_{\mathbb{R}_{0}^{+}}(r) .
$$

Observe that both (16) and (17) are extended by continuity when $M_{2}=M_{1}$, since:

$$
\begin{aligned}
& \lim _{M_{2} \rightarrow M_{1}} F_{R}\left(r ; \mathbf{M}_{2}, 0\right)=\left(1-\frac{2 \log 2 M_{1} r+1}{2^{2 M_{1} r}}\right) \mathbb{1}_{\mathbb{R}_{0}^{+}}(r), \\
& \lim _{M_{2} \rightarrow M_{1}} f_{R}\left(r ; \mathbf{M}_{2}, 0\right)=2 \log ^{2} 2 M_{1}^{2} r 2^{-2 M_{1} r} \mathbb{1}_{\mathbb{R}_{0}^{+}}(r) .
\end{aligned}
$$

The formulas for $L \geq 3$ are obtained by iterating the integration process. In fact, once the pdf $f_{R}\left(r ; \mathbf{M}_{L-1}, 0\right)$ in the presence of $L-1$ segments has been evaluated, the cdf of 


$$
\begin{aligned}
& F_{R}\left(r ; \mathbf{M}_{L}, \varsigma\right)=\int_{-\infty}^{+\infty} \ldots \int_{-\infty}^{+\infty}\left[F_{\Lambda_{L} \mid \boldsymbol{\Lambda}_{L-1}}\left(L r-\sum_{l=1}^{L-1} \lambda_{l} \mid \boldsymbol{\lambda}_{L-1} ; \mathbf{M}_{L}, \varsigma\right) \prod_{l=1}^{L-1} f_{\Lambda_{l} \mid \boldsymbol{\Lambda}_{l-1}}\left(\lambda_{l} \mid \boldsymbol{\lambda}_{l-1} ; \mathbf{M}_{l}, \varsigma\right)\right] \mathrm{d} \lambda_{1} \ldots \mathrm{d} \lambda_{L-1} \\
& f_{X_{l} \mid S_{0}}\left(x_{l} \mid s_{0} ; M_{l}, \varsigma\right)=\left\{\frac{s_{0}}{\zeta^{M_{l}}\left(M_{l}-1\right) ! x_{l}^{2}}\left(\frac{s_{0}}{x_{l}}-\varsigma\right)^{M_{l}-1} \exp \left[\frac{1}{\zeta}\left(\varsigma-\frac{s_{0}}{x_{l}}\right)\right]\right\} \mathbb{1}_{\mathbf{A}_{l}^{\varsigma}}\left(x_{l}, s_{0}\right) \\
& f_{S_{0} \mid \mathbf{X}_{l-1}}\left(s_{0} \mid \mathbf{x}_{l-1} ; \mathbf{M}_{l-1}, \varsigma\right)=\frac{f_{X_{l-1} \mid S_{0}}\left(x_{l-1} \mid s_{0} ; M_{l-1}, \varsigma\right) f_{S_{0} \mid \mathbf{X}_{l-2}}\left(s_{0} \mid \mathbf{x}_{l-2} ; \mathbf{M}_{l-2}, \varsigma\right)}{f_{X_{l-1} \mid \mathbf{X}_{l-2}}\left(x_{l-1} \mid \mathbf{x}_{l-2} ; \mathbf{M}_{l-1}, \varsigma\right)} \\
& f_{X_{l} \mid \mathbf{x}_{l-1}}\left(x_{l} \mid \mathbf{x}_{l-1} ; \mathbf{M}_{l}, 0\right)=\frac{\mathcal{M}_{l} !}{\mathcal{M}_{l-1} !\left(M_{l}-1\right) !}\left(1+\sum_{l^{\prime}=1}^{l-1} \frac{1}{x_{l^{\prime}}}\right)^{\mathcal{M}_{l-1}+1}\left[x_{l}\left(1+\sum_{l^{\prime}=1}^{l} \frac{1}{x_{l^{\prime}}}\right)\right]^{-\mathcal{M}_{l}-1} \mathbb{1}_{\mathbf{B}_{l}}\left(\mathbf{x}_{l}\right) \\
& F_{X_{l} \mid \mathbf{X}_{l-1}}\left(x_{l} \mid \mathbf{x}_{l-1} ; \mathbf{M}_{l}, 0\right)=\frac{(-1)^{\mathcal{M}_{l-1}+1} \mathcal{M}_{l} !}{\mathcal{M}_{l-1} !\left(M_{l}-1\right) !} \beta\left[-\left(1+\sum_{l^{\prime}=1}^{l-1} \frac{1}{x_{l^{\prime}}}\right) x_{l} ; \mathcal{M}_{l-1}+1,-\mathcal{M}_{l}\right] \mathbb{1}_{\mathbf{B}_{l}}\left(\mathbf{x}_{l}\right)
\end{aligned}
$$

$R$ in the presence of $L$ segments can be calculated performing just another integration. This yields:

$$
\begin{aligned}
F_{R}\left(r ; \mathbf{M}_{L}, 0\right)= & \frac{1}{L-1} \int_{0}^{L r}\left[f_{R}\left(\frac{\lambda_{L-1}}{L-1} ; \mathbf{M}_{L-1}, 0\right)\right. \\
& \left.\cdot F_{\Lambda_{L}}\left(L r-\lambda_{L-1} ; M_{L}, 0\right)\right] \mathrm{d} \lambda_{L-1} \\
= & \left(1-\sum_{l=1}^{L} 2^{-L M_{l} r} \prod_{\substack{l^{\prime}=1 \\
l^{\prime} \neq l}}^{L} \frac{M_{l^{\prime}}}{M_{l^{\prime}}-M_{l}}\right) \mathbb{1}_{\mathbb{R}_{0}^{+}}(r),
\end{aligned}
$$

from which the corresponding pdf is obtained as:

$$
f_{R}\left(r ; \mathbf{M}_{L}, 0\right)=\log 2 \sum_{l=1}^{L} \frac{L M_{l}}{2^{L M_{l} r}} \prod_{\substack{l^{\prime}=1 \\ l^{\prime} \neq l}}^{L} \frac{M_{l^{\prime}}}{M_{l^{\prime}}-M_{l}} \mathbb{1}_{\mathbb{R}_{0}^{+}}(r) .
$$

Also (20) and (21) can be extended by continuity to include the cases in which two or more $M_{l}$ values become identical. The capture probability for the fast scenario in an interference context $\mathbf{M}_{L}$ may be then derived, for the specific interferencelimited case, by inserting (20) in (12), while, for the general case, by numerically evaluating (13) and then using (12).

\section{B. Slow fading scenario}

In the slow fading scenario, the $L$ r.v.s $\Lambda_{1}, \ldots, \Lambda_{L}$ are dependent. In fact, the SINRs $X_{1}, \ldots, X_{L}$ are correlated, since the power received from the target user is the same for all the $L$ segments. The cdf of $R$ has to be hence evaluated by accounting for the conditioning between the SINRs of the different segments belonging to a given packet. Therefore, (13) must be generalized according to (22), in which, for $l=1, \ldots, L$, the random vector of the first $l$ rates $\boldsymbol{\Lambda}_{l}=$ $\left[\Lambda_{1}, \ldots, \Lambda_{l}\right]$ and the corresponding realization $\lambda_{l}=\left[\lambda_{1}, \ldots, \lambda_{l}\right]$ are introduced to obtain a compact notation, and $\boldsymbol{\Lambda}_{0}=\boldsymbol{\lambda}_{0}=\emptyset$ is defined for mathematical purposes.
To analyze this situation, the pdf of $X_{l}$ given $\mathbf{X}_{l-1}(l=$ $1, \ldots, L)$ must be first evaluated by solving the integral:

$$
\begin{array}{r}
f_{X_{l} \mid \mathbf{x}_{l-1}}\left(x_{l} \mid \mathbf{x}_{l-1} ; \mathbf{M}_{l}, \varsigma\right)=\int_{-\infty}^{+\infty}\left[f_{X_{l} \mid S_{0}}\left(x_{l} \mid s_{0} ; M_{l}, \varsigma\right)\right. \\
\left.\cdot f_{S_{0} \mid \mathbf{X}_{l-1}}\left(s_{0} \mid \mathbf{x}_{l-1} ; \mathbf{M}_{l-1}, \varsigma\right)\right] \mathrm{d} s_{0}
\end{array}
$$

in which $f_{X_{l} \mid S_{0}}\left(x_{l} \mid s_{0} ; M_{l}, \varsigma\right)$ is the pdf of $X_{l}$ given $S_{0}$, $f_{S_{0} \mid \mathbf{X}_{l-1}}\left(s_{0} \mid \mathbf{x}_{l-1} ; \mathbf{M}_{l-1}, \varsigma\right)$ is the pdf of $S_{0}$ given $\mathbf{X}_{l-1}$, with $\mathbf{X}_{l}=\left[X_{1}, \ldots, X_{l}\right], \mathbf{x}_{l}=\left[x_{1}, \ldots, x_{l}\right]$ for $l=1, \ldots, L$, and $\mathbf{X}_{0}=\mathbf{x}_{0}=\emptyset$. Recalling (4), the pdf of $X_{l}$ given $S_{0}$ for $l=1, \ldots, L$ is calculated from:

$$
f_{X_{l} \mid S_{0}}\left(x_{l} \mid s_{0} ; M_{l}, \varsigma\right)=-\frac{\mathrm{d}}{\mathrm{d} x_{l}}\left[F_{U_{l}}\left(\frac{s_{0}}{x_{l}} ; M_{l}, \varsigma\right)\right],
$$

which provides (25), where $\mathbf{A}_{l}^{\varsigma}=\left\{\left(x_{l}, s_{0}\right) \mid 0<x_{l} \leq\right.$ $\left.s_{0} / \varsigma, s_{0} \geq 0\right\}$. The pdf of $S_{0}$ given $\mathbf{X}_{l-1}$ is instead derived, for $l=2, \ldots, L$, from the Bayes formula according to (26). In particular, the first case in (26), corresponding to $l=2$, is analyzed by using (2) for $m=0$, and (6) and (25) for $l=1$. The resulting expression is then inserted in (23) to derive $f_{X_{2} \mid X_{1}}\left(x_{2} \mid x_{1} ; \mathbf{M}_{2}, \varsigma\right)$. These two operations may be viewed as the first step of an iterative process, since (26) and (23) can be iteratively applied to obtain $f_{X_{l} \mid \mathbf{X}_{l-1}}\left(x_{l} \mid \mathbf{x}_{l-1} ; \mathbf{M}_{l}, \varsigma\right)$ for any $l$ value, until all the $L$ segments are considered.

The above described procedure does not provide closedform expressions for any $\varsigma$ value, and hence, in general, numerical techniques must be adopted. However, an interesting analytical formula is again derived for the interference-limited case. In fact, for $\varsigma$ approaching zero and defining the set $\mathbf{B}_{l}=\left\{\mathbf{x}_{l} \mid x_{l^{\prime}}>0, l^{\prime}=1, \ldots, l\right\}$, the iteration of (26) and (23) until $l$ provides (27), where $\mathcal{M}_{l}=\sum_{l^{\prime}=1}^{l} M_{l^{\prime}}$. Once (27) is available, the corresponding cdf is obtained as:

$$
F_{X_{l} \mid \mathbf{X}_{l-1}}\left(x_{l} \mid \mathbf{x}_{l-1} ; \mathbf{M}_{l}, \varsigma\right)=\int_{-\infty}^{x_{l}} f_{X_{l} \mid \mathbf{X}_{l-1}}\left(x_{l}^{\prime} \mid \mathbf{x}_{l-1} ; \varsigma\right) \mathrm{d} x_{l}^{\prime},
$$

which, in the interference-limited case, has the analytical form in (29), where $\beta(\cdot ; \cdot, \cdot)$ is the incomplete beta function [22].

Now, remembering the relationship between the rate $\Lambda_{l}$ and the SINR $X_{l}$ in (7), one can express the conditional cdf and 


$$
\bar{c}_{n}(\varrho ; \varsigma)=(n-1) ! \sum_{n_{1}=t_{1}(\mathbf{n})}^{T_{1}(\mathbf{n})} \ldots \sum_{n_{L+1}=t_{L+1}(\mathbf{n})}^{T_{L+1}(\mathbf{n})} \frac{p_{1}^{n_{1}} \cdots p_{L+1}^{n_{L+1}}}{n_{1} ! \cdots n_{L+1} !} c\left[\varrho ; \mathbf{M}_{L}(\mathbf{n}), \varsigma\right]
$$

pdf of $\Lambda_{l}$, respectively, as:

$F_{\Lambda_{l} \mid \mathbf{\Lambda}_{l-1}}\left(\lambda_{l} \mid \boldsymbol{\lambda}_{l-1} ; \mathbf{M}_{l}, \varsigma\right)=F_{X_{l} \mid \mathbf{X}_{l-1}}\left(2^{\lambda_{l}}-1 \mid \mathbf{z}_{l-1} ; \mathbf{M}_{l}, \varsigma\right)$,

$f_{\Lambda_{l} \mid \boldsymbol{\Lambda}_{l-1}}\left(\lambda_{l} \mid \boldsymbol{\lambda}_{l-1} ; \mathbf{M}_{l}, \varsigma\right)=\frac{\log 2}{2^{-\lambda_{l}}} f_{X_{l} \mid \mathbf{x}_{l-1}}\left(2^{\lambda_{l}}-1 \mid \mathbf{z}_{l-1} ; \mathbf{M}_{l}, \varsigma\right)$,

where $\mathbf{z}_{l}=\left[2^{\lambda_{1}}-1, \ldots, 2^{\lambda_{l}}-1\right]$. These two statistics can be inserted in (22) to derive the cdf of $R$, from which, by (12), one finally obtains the capture probability in an interference context $\mathbf{M}_{L}$. Specific closed-forms may be derived for $\varsigma=0$, such as for $L=2$ and $\mathbf{M}_{L}=[1,1]$, which provide $F_{R}(r ;[1,1], 0)=$ $\left\{1-2\left[1-2^{2 r}(1+2 \log 2 r)\right] /\left(2^{2 r}-1\right)^{2}\right\} \mathbb{1}_{\mathbb{R}_{0}^{+}}(r)$. Observe that, in general, the availability of closed-forms for $\varsigma=0$ is relevant, since, in the presence of multiple contending nodes sharing a common medium, the current satellite and cellular networks behave as interference-limited systems.

\section{Throughrut ANALYSIS}

Once the statistics of the equivalent rate are available, the throughput analysis is carried out following two main steps. Firstly, the average capture probability in the presence of $n$ $(1 \leq n \leq N)$ contending users is estimated. Secondly, a Markov chain approach is developed to model the system evolution, so as to obtain the number of captured packets, and, in turn, the network throughput in the presence of IC.

\section{A. Average capture probability}

The instantaneous channel load for an FSA system with $N$ users transmitting their packets in a RAF consisting of $K$ slots is defined, according to [7-9], as:

$$
G=\varepsilon \frac{N}{K}=\varepsilon \sigma
$$

where $0 \leq \varepsilon \leq 1$ is the activation probability and $\sigma$ is the normalized user population size. Still in agreement with [79], it is assumed that each user has always a packet ready for transmission in its buffer (saturated traffic condition), and that retransmissions are not allowed. Thus, for an individual packet, just one transmission, carried out with probability $\varepsilon$, is attempted. If this transmission is unsuccessful, the packet is discarded and the user gets another one from its transmission buffer. Assuming the users' activity as binomially distributed, the probability that $n$ packets are transmitted, that is, $n$ users become active, is given by:

$$
\tau_{n}=\left(\begin{array}{c}
N \\
n
\end{array}\right) \varepsilon^{n}(1-\varepsilon)^{N-n} .
$$

Among these $n$ active users, consider the so far addressed target user and another given user, both transmitting the $L$ segments of their packets in the same RAF of $K \cdot L$ slices. To analyze this situation one has to identify, for the given user and for $j=1, \ldots, L+1$, the event represented by the transmission of $j-1$ segments in slices different from those selected by the target user and of $L-(j-1)$ segments in slices identical to those selected by the target user. In particular, this event is characterized, for $j=1, \ldots, L+1$, by the probability:

$$
p_{j}=\frac{\left(\begin{array}{c}
L \\
L-j+1
\end{array}\right)\left(\begin{array}{c}
K L-L \\
j-1
\end{array}\right)}{\left(\begin{array}{c}
K L \\
L
\end{array}\right)}
$$

that it occurs, and by the number of users $n_{j}$ experiencing it, with $\sum_{j=1}^{L+1} n_{j}=n-1$. One may then notice that, when $\varrho$ and $\varsigma$ are given, $c\left(\varrho ; \mathbf{M}_{L}, \varsigma\right)$ remains the same for any permutation of $\mathbf{M}_{L}$, thus its components can be arranged in non-descending order without loss of generality. Therefore, defining the vector $\mathbf{n}=\left[n_{1}, \ldots, n_{L+1}\right]$, the interference context of the target user becomes $\mathbf{M}_{L}(\mathbf{n})=\left[M_{1}(\mathbf{n}), \ldots, M_{L}(\mathbf{n})\right]$, where the collision order for its $l$-th segment is given by the partial sum:

$$
M_{l}(\mathbf{n})=\sum_{j=1}^{l} n_{j}, \quad l=1, \ldots, L
$$

Hence, considering all the possible combinations of the number of interferers in the $L$ slices by introducing the multinomial coefficient, the average capture probability for the target user when other $n-1$ contending users are present is calculated by (36), where the lower and upper bounds of the summations are defined, respectively, by:

$$
\begin{array}{r}
t_{j}(\mathbf{n})= \begin{cases}0 & j=1, \ldots, L \\
N-1-M_{L}(\mathbf{n}) & j=L+1\end{cases} \\
T_{j}(\mathbf{n})= \begin{cases}N-1 & j=1 \\
N-1-M_{j-1}(\mathbf{n}) & j=2, \ldots, L+1\end{cases}
\end{array}
$$

The quantity in (36) enables to derive the probability of capturing $n^{\prime}$ over $n$ packets as:

$$
\alpha_{n, n^{\prime}}(\varrho ; \varsigma)=\left(\begin{array}{c}
n \\
n^{\prime}
\end{array}\right) \bar{c}_{n}^{n^{\prime}}(\varrho ; \varsigma)\left[1-\bar{c}_{n}(\varrho ; \varsigma)\right]^{n-n^{\prime}}
$$

by assuming a binomial distribution for the success/failure capture occurrences. This estimation represents the first step for evaluating the throughput at the generic iteration $i$. Note that the term iteration is here intended as the combination of a capture cycle, first, and of an IC one, subsequently. For example, when $i=1$, the first capture cycle is carried out, while the subsequent IC cycle is assumed to be formally accomplished, but exploited just if a second iteration is allowed. Thus, one obtains the throughput of an FSA system with segmentation and capture (one cycle) for $i=1$, and with segmentation, capture ( $i$ cycles), and IC ( $i-1$ cycles) for $i>1$. 


$$
T_{t, h, t^{\prime}, h^{\prime}}^{(n)}(\varrho ; \varsigma)=\left\{\begin{array}{lll}
\alpha_{n-t, n-t^{\prime}}(\varrho ; \varsigma) & 0 \leq t<n, t+1 \leq t^{\prime}<n, & h=h^{\prime}=0 \\
\alpha_{n-t, n-t}(\varrho ; \varsigma) & 0 \leq t<n, t^{\prime}=n, & h=1-h^{\prime}=0 \\
\alpha_{n-t, 0}(\varrho ; \varsigma) & 0 \leq t<n, t^{\prime}=t, & h=1-h^{\prime}=0 \\
1 & 0 \leq t \leq n, t^{\prime}=t, & h=h^{\prime}=1 \\
0 & \text { otherwise }
\end{array}\right.
$$

\section{B. Throughput}

Consider now, as a second step of the throughput analysis, the evolution of the system through a family of $N$ twodimensional Markov chains, where each chain describes the system evolution in the presence of $n$ users. To this aim, define the generic state of a chain as $(t, h)$, where $t(0 \leq$ $t \leq n)$ denotes the number of captured packets, and $h$ specifies if the decoding procedure is in progress $(h=0)$ or is terminated $(h=1)$. Concerning this second index, observe that the decoding process is stopped when, after an IC cycle, no novel captures occur. Accordingly, for the specific chain modeling the system with $n$ packets present in the RAF, the transition probabilities from a state $(t, h)$ to a state $\left(t^{\prime}, h^{\prime}\right)$ are calculated by (40). Accounting for all the possible $n$ values, these probabilities are jointly viewed as the elements of an indexed family of $N$ four-dimensional transition matrices $\left\{\mathbf{T}^{(n)}(\varrho ; \varsigma)\right\}_{n=1}^{N}$, where the generic element of the family $\mathbf{T}^{(n)}(\varrho ; \varsigma)=\left[T_{t, h, t^{\prime}, h^{\prime}}^{(n)}(\varrho ; \varsigma)\right] \in[0,1]^{(n+1) \times 2 \times(n+1) \times 2}$ is plane-stochastic along the first two dimensions, that is, $\sum_{t=0}^{n} \sum_{h=0}^{1} T_{t, h, t^{\prime}, h^{\prime}}^{(n)}(\varrho ; \varsigma)=1$ for $t^{\prime}=0, \ldots, n$ and $h^{\prime}=0,1$. The probability of being in the state $(t, h)$ at the $i$-th iteration when $n$ packets are present in the RAF can be denoted as $\pi_{t, h}^{(i, n)}(\varrho ; \varsigma)$, which represents the generic element of the state matrix $\pi^{(i, n)}(\varrho ; \varsigma)=\left[\pi_{t, h}^{(i, n)}(\varrho ; \varsigma)\right] \in[0,1]^{(n+1) \times 2}$. The evolution of the $n$-th chain starts from the state $(0,0)$ and from $i=0$, corresponding to none iteration yet performed, thus:

$$
\pi_{t, h}^{(0, n)}(\varrho ; \varsigma)= \begin{cases}1 & t=h=0 \\ 0 & \text { otherwise }\end{cases}
$$

while, for $i \geq 1$, the probability of moving from a state $(t, h)$ at the $(i-1)$-th iteration to a state $\left(t^{\prime}, h^{\prime}\right)$ at the $i$-th iteration is calculated as:

$$
\begin{aligned}
\pi_{t^{\prime}, h^{\prime}}^{(i, n)}(\varrho ; \varsigma) & =\sum_{t=0}^{n} \sum_{h=0}^{1} T_{t, h, t^{\prime}, h^{\prime}}^{(n)}(\varrho ; \varsigma) \pi_{t, h}^{(i-1, n)}(\varrho ; \varsigma) \\
& =\left\langle\mathbf{T}_{t^{\prime}, h^{\prime}}^{(n)}(\varrho ; \varsigma), \boldsymbol{\pi}^{(i-1, n)}(\varrho ; \varsigma)\right\rangle_{\mathrm{F}},
\end{aligned}
$$

where $\mathbf{T}_{t^{\prime}, h^{\prime}}^{(n)}(\varrho ; \varsigma) \in[0,1]^{(n+1) \times 2}$ is the two-dimensional submatrix of $\mathbf{T}^{(n)}(\varrho ; \varsigma)$ corresponding to the pair $\left(t^{\prime}, h^{\prime}\right)$, and $\langle\cdot, \cdot\rangle_{\mathrm{F}}$ denotes the Frobenius inner product. Introducing the symbol $\odot$ to identify this latter operation for all possible $\left(t^{\prime}, h^{\prime}\right)$ pairs, one may jointly express all the state probabilities at the $i$-th iteration when $n$ users are present as:

$$
\boldsymbol{\pi}^{(i, n)}(\varrho ; \varsigma)=\mathbf{T}^{(n)}(\varrho ; \varsigma) \odot \boldsymbol{\pi}^{(i-1, n)}(\varrho ; \varsigma) .
$$

Moving from the starting point $\boldsymbol{\pi}^{(0, n)}(\varrho ; \varsigma)$ defined in (41), this latter relationship allows one to iteratively derive all the state matrices at all iterations for $n=1, \ldots N$. The availability of each $\boldsymbol{\pi}^{(i, n)}(\varrho ; \varsigma)$ matrix when $n$ users at iteration $i$ are present enables the evaluation of the average number of packets captured until iteration $i$ as:

$$
\mathcal{C}^{(i, n)}(\varrho ; \varsigma)=\sum_{t=1}^{n} t\left[\pi_{t, 0}^{(i, n)}(\varrho ; \varsigma)+\pi_{t, 1}^{(i, n)}(\varrho ; \varsigma)\right],
$$

which, by (33), finally allows the estimation of the system throughput until the $i$-th iteration as:

$$
\eta_{i}(\varrho ; \varsigma)=\frac{\varrho}{K} \sum_{n=0}^{N} \tau_{n} \mathcal{C}^{(i, n)}(\varrho ; \varsigma),
$$

where the transmission probability $\tau_{n}$ is defined in (33).

Observe that the developed multi-dimensional Markov model involving both $n$ and $i$, even if elaborated, is necessary to include all the possible system evolutions. In fact, a univocal relationship between the iteration and the number of packets captured until that iteration does not exist, thus all possible $n$ values must be considered at each iteration. This is the reason for the definition of the families of the four-dimensional transition matrices in (40) and of the state probabilities in (43). Concerning the presented theoretical model, it is also worth to notice that the main computational cost for its implementation is due to the estimation of the capture probability. In particular, the number of elements of the family of Markov chains that have to be considered for the evaluation of the throughput increases with $N$, which also influences the size of the largest chains of the family. However, the subsequent derivation of the state probabilities simply requires products between fourdimensional matrices, whose complexity grows as $O\left(N^{4}\right)$. The complexity of the capture evaluation, instead, depends not only on $N$, but also on $K, L$, and the fading model. Concerning $c\left(\varrho ; \mathbf{M}_{L}, \varsigma\right)$ in (12), the evaluation results simpler in the fast fading scenario, thanks to the independence among the $L$ rates $\lambda_{1}, \ldots, \lambda_{L}$, while, in the slow fading scenario, the estimation requires much more efforts. In this second case, in fact, the $L$ rates are no more independent and the cdf of $R$, as proved in Subsection III-B, must be derived by $L$ recursive sets of operations, characterized by a computational complexity that grows as $O(L)$. Concerning $\bar{c}_{n}(\varrho ; \varsigma)$ in (36), its estimation is instead a problem of exponential complexity that grows with $N, K$, and $L$ as $O\left[(K L)^{N}\right]$.

As a final remark, it is worth to observe that the main conceptual difference between the here developed analysis and the previously proposed ones lies in the receiving model. In [9], in fact, the analysis relies on a decoupled approach in which the reception is verified for one segment at a time by checking that it lies in a clean (or cleaned after IC) slice (single-segment erasure model). Instead, in the here proposed framework, capture is implemented by adopting a collective approach that jointly considers the SINR of all segments (jointsegments capture model). From the strict computational point 


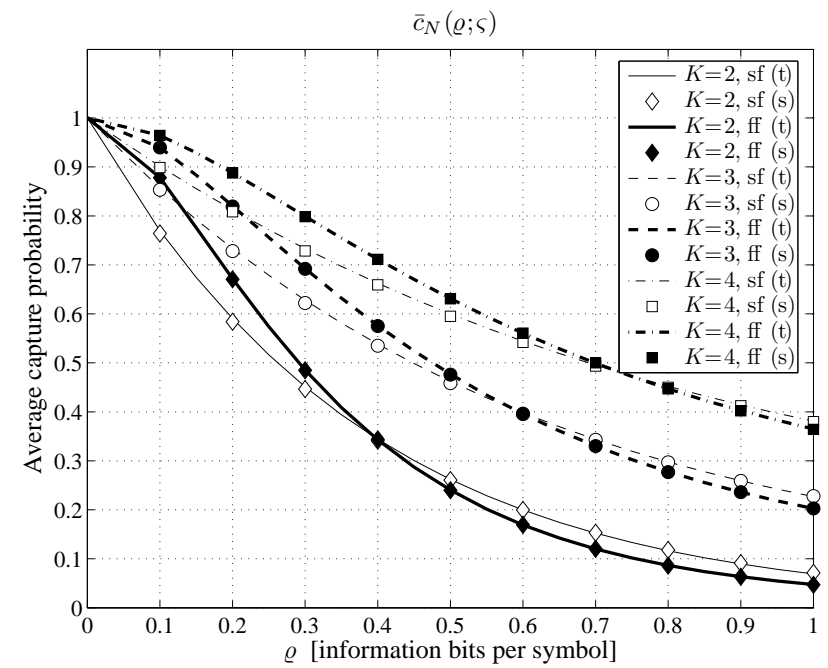

(a)

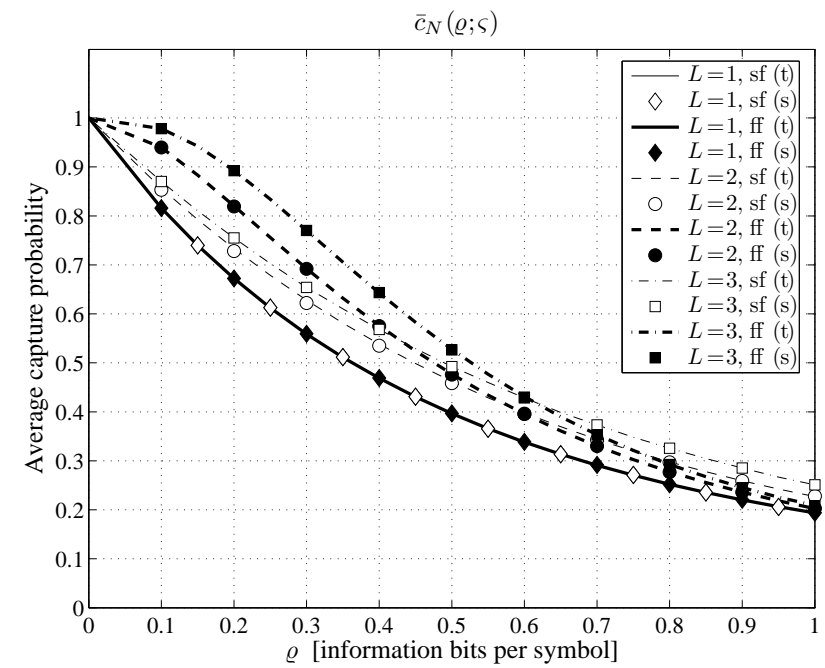

(b)

Fig. 1. Average capture probability as a function of the selected rate in slow fading (sf) and fast fading (ff) scenarios (t: theory, s: simulation): (a) $L=2$ and different number of slots, (b) $K=3$ and different number of segments.

of view, this leads, with respect to the previous theories [9], to a problem that can be formally developed for any triad $(N, K, L)$, even if its actual implementation is limited by an exponential cost. This, however, may represent an unavoidable difficulty to deal with, if a realistic capture model for a segmented-FSA system has to be adopted, since even the most degraded segment might provide a residual contribution determinant for overcoming the reception threshold.

\section{RESUlts}

This section presents the derived numerical results by subdividing the discussion into three parts. The first one focuses on the case $i=1$, thus considering the capture probability and the throughput of an FSA system with segmentation and capture in the absence of IC. The second part analyzes also the cases $i>1$ by investigating the impact of IC and segmentation at the different iterations. These two parts consider the performance achievable through the Shannon bound, and include, for all the analyzed scenarios, both the theoretical and simulated curves. The third part examines the conceived decoding criterion through a practical communication system adopting a QPSK modulation combined with a turbo encoder.

All theoretical and practical results, which are obtained for $\zeta=0 \mathrm{~dB}$ and $\varsigma=-30 \mathrm{~dB}$, are compared to independent Monte Carlo simulations, where each point of a simulated curve is obtained by averaging over $Q=10000$ realizations, that is, over $Q$ RAFs. Subsequent RAFs experience independent fading levels, while, within a RAF, the scenario can evolve according to each of those addressed in Section III. In particular, for a given user and a given RAF, the fading level is constant for all segments in the slow fading scenario, while different segments have independent fading levels in the fast fading one.

\section{A. FSA with segmentation and capture}

The first set of results is shown in Fig. 1, which reports the average capture probability $\bar{c}_{n}(\varrho ; \varsigma)$ in (36) as a function of

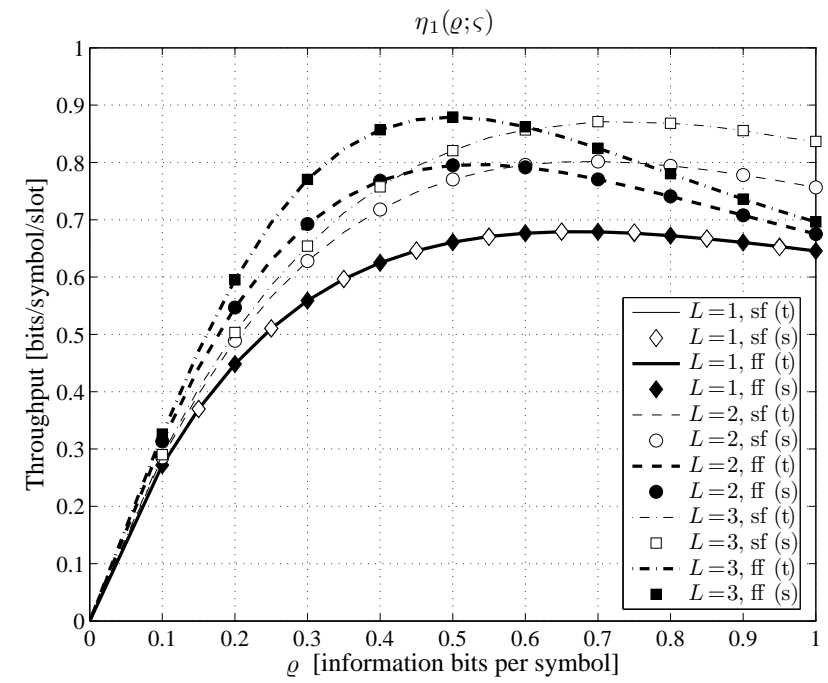

Fig. 2. Throughput as a function of the selected rate in slow fading (sf) and fast fading (ff) scenarios for $K=3$ and different number of segments ( $\mathrm{t}$ : theory, s: simulation).

the selected rate for $n=N$. The system is hence observed at the beginning of the decoding process, when no captures have even occurred and all transmitted packets are still in the RAF. In particular, the results are obtained for $N=10$ in the fast and slow fading scenarios considering $L=2$ segments and different number of slots (Fig. 1(a)), and $K=3$ slots and different number of segments (Fig. 1(b)). The theoretical curves are identified by lines, while those derived from Monte Carlo simulations, which present an excellent matching with the theory, are identified by markers.

The figure shows that, when $K$ and $L(>1)$ are fixed, the slow and fast fading scenarios lead to a close performance, being the capture probability in fast fading conditions slightly higher than that in the slow fading ones until the code rate 


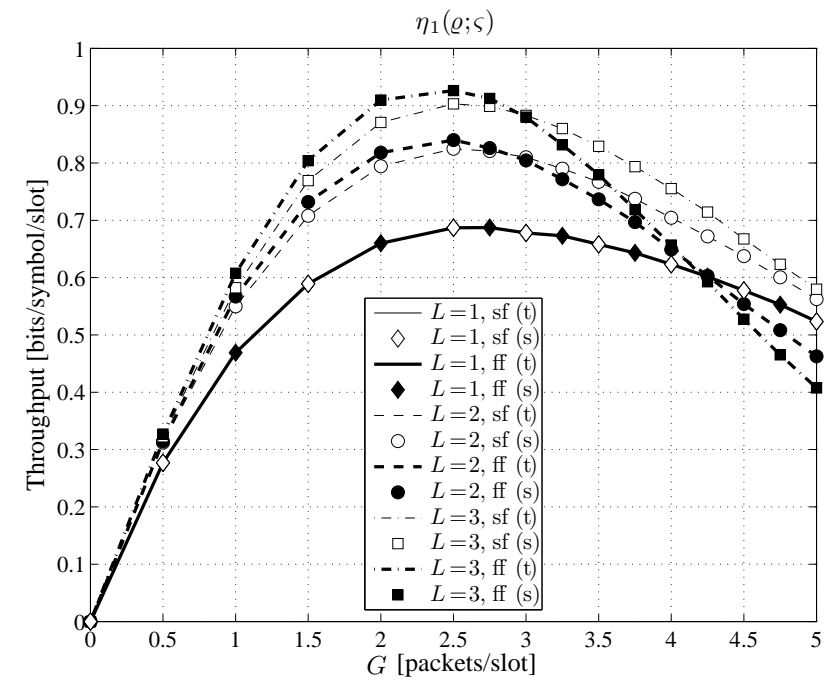

Fig. 3. Throughput as a function of the offered load in slow fading (sf) and fast fading (ff) scenarios for $K=3, \sigma=5$ users/slot, and different number of segments (t: theory, s: simulation).

does not become close to one. This is due to the beneficial power diversity effect present in the fast fading case, which increases the probability that one of the powers of the segments colliding in a slice is sufficiently higher than the sum of the powers of the other segments present in the same slice. Of course, the two scenarios coincide when segmentation is not applied $(L=1)$. As expected, the increase of $K$ increases $\bar{c}_{N}(\varrho ; \varsigma)$ for all rates, since the duration of the RAF becomes longer and the average interference in each slice decreases. This increase of the capture probability for higher $K$ values obtained using the developed decoding criterion is analogous to the corresponding reduction of the collision probability obtained when the erasure channel is employed [7-9].

Benefits on the FSA performance are obtained by segmentation, since an increase of $L$ provides an increase of the capture probability for all the considered rates in fast and slow fading scenarios (Fig. 1(b)). This behavior is confirmed by Fig. 2, which reports the throughput corresponding to the six cases of Fig. 1(b) and reveals the existence of an optimal $\varrho$ value dependent on the scenario and on the number of segments and slots. More precisely, one may observe that, in fast fading scenarios, the usage of higher $L$ values leads to a reduction of the optimal rate, thus suggesting a more conservative selection of the transmission rate, with lower order modulations and more powerful codes, as the number of segments increases. Conversely, in slow fading scenarios, the optimal $\varrho$ value seems almost insensitive to segmentation. Hence, in this case, a unique rate can be selected for different $L$ values.

Still concerning Fig. 1, one may notice that the increase of $K$ and $L$ improves the capture probability, even if the two quantities act differently. To explain this aspect, consider a specific fading scenario with a given number of transmitters. In this situation, when $L$ is fixed, the increase of $K$ statistically leads, in each slice, to a lower load, and, in turn, to a lower interference, that increases $\bar{c}_{N}(\varrho ; \varsigma)$. Instead, when $K$ is fixed, the increase of $L$ does not change the size of the RAF and hence no statistical reductions of the interference occur. Rather, a larger $L$ value leads to a higher differentiation of the interference suffered by the distinct segments of a packet, which leads to an interference diversity among the slices occupied by those segments. Observe that this benefit, even if less pronounced than that provided by an increment of the number of slots (Fig. 1), has the considerable advantage of not increasing the packet delay due to the contention process, since the segmentation technique does not modify the RAF duration.

As a final result for this subsection, Fig. 3 reports the throughput as a function of the offered load for the six cases of Fig. 1(b). The curves are derived for $K=3$ and using the rate $\varrho=2 / 3$, which represents a reasonable choice providing a satisfactory performance for both slow and fast fading scenarios (Fig. 2). For this reason the value $\varrho=2 / 3$ will be adopted from now on to evaluate the results. Besides, to consider a scenario in which the number of users is large with respect to the number of available slots, so as to properly check the performance of a random access scheme [9], the parameter $\sigma$ is assumed fixed and equal to 5 users per slot, while the activation probability $\varepsilon$ is gradually increased from 0 to 1 . Also this setting will be used for the sequel of this section. Fig. 3 shows the existence of an optimal $G$ value, corresponding to an optimal activation probability that maximizes the throughput. Furthermore, the figure reveals that, in a fast fading scenario, segmentation may be not advantageous when the offered load is very high. However, this is a limiting situation, corresponding to an activation probability approaching one, in which a demand assignment access protocol can be more suitable than a random access one [9].

\section{B. FSA with segmentation, capture, and IC}

This second subsection discusses the impact of IC, and hence of the iteration, on the FSA performance. A first group of results is reported in Fig. 4, which shows the throughput as a function of the offered load at different iterations for $L=2$. In particular, Fig. 4(a) refers to a slow fading scenario with $K=2$, Fig. 4(b) to a fast fading scenario with $K=2$, Fig. 4(c) to a slow fading scenario with $K=3$, and Fig. 4(d) to a fast fading scenario with $K=3$. In the legends, the notation $i=N$ indicates, for the theoretical curves, the maximum number of iterations that are performed, while, for the simulated curves, it has just a formal meaning, since it indicates that the system is left to evolve until captures are possible after an IC cycle, thus leading to a maximum achieved iteration that can be different from realization to realization.

The figure shows that, for a fixed $K$ value, the performance of the fast and slow fading scenarios remain very close also when the higher iterations are taken into account, thus confirming the behavior already observed in the previous set of results. Besides, as expected, IC considerably improves the performance, which gets higher as the number of allowed iterations is increased. It is interesting to put into evidence that also the benefits provided by the increase of the number slots become more clear when more iterations are allowed. To outline this issue, compare the cases $K=2$ and $K=3$ 


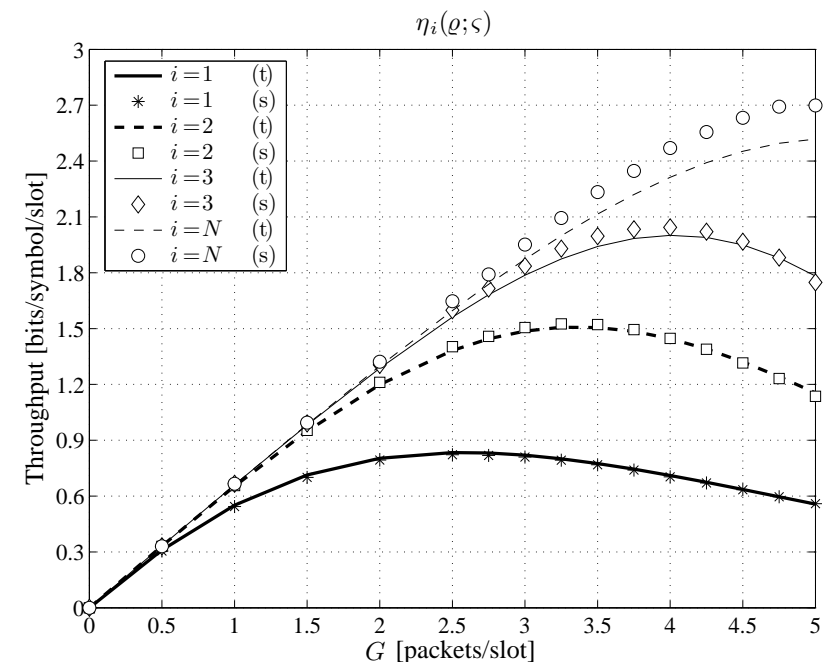

(a)

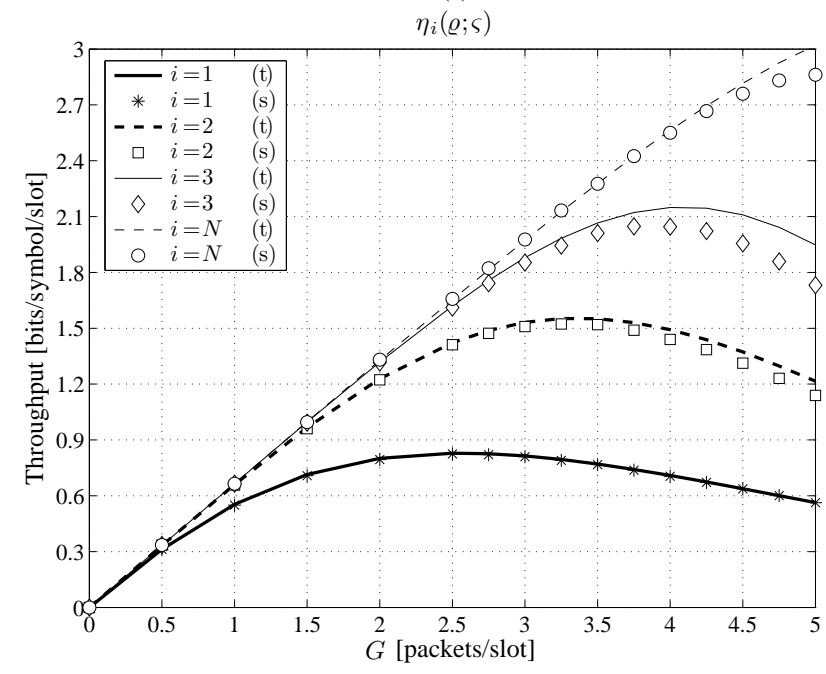

(c)

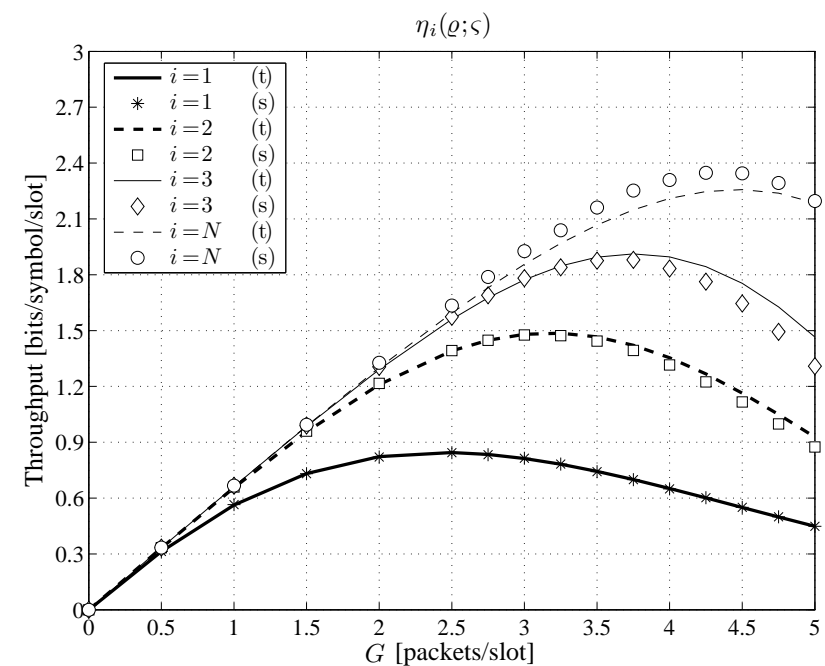

(b)

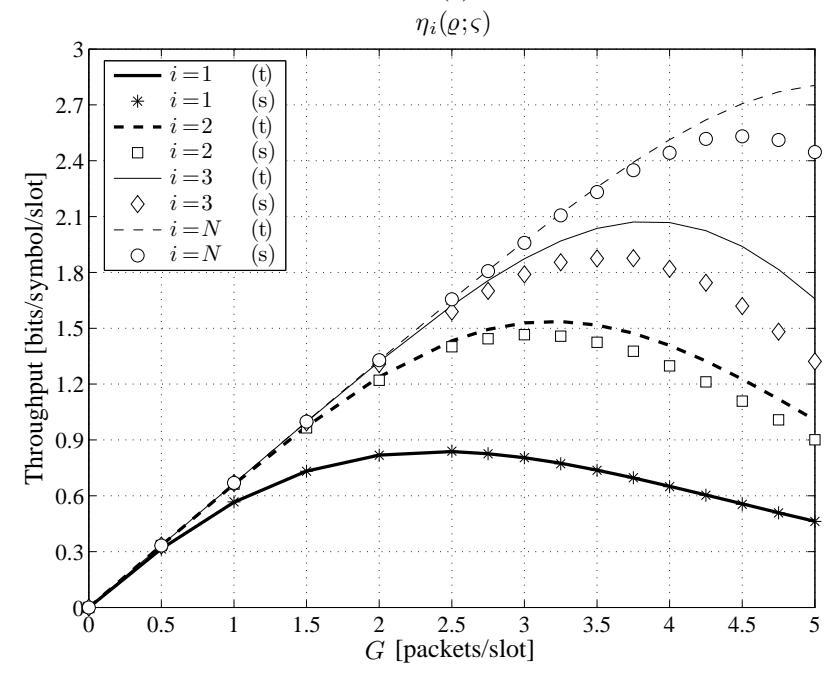

(d)

Fig. 4. Throughput as a function of the offered load at different iterations for $L=2$ (t: theory, s: simulation): (a) $K=2$ in slow fading scenario, (b) $K=2$ in fast fading scenario, (c) $K=3$ in slow fading scenario, (d) $K=3$ in fast fading scenario.

for a given fading scenario, and notice that, for $i=1,2,3$, the corresponding curves for different number of slots are very similar, while an observable increase of the throughput is noticed for $i=N$. This suggests that the FSA system should be left to evolve until captures occur, that is, allowing all the IC cycles necessary to exploit a longer RAF duration.

The requirement of considering many IC cycles to obtain an appreciable performance improvement when $K$ is increased is instead not necessary when the increase involves $L$. To better observe this aspect, consider Fig. 5, which reports, for a fast fading scenario and for $L=3$, the throughput as a function of the offered load at the different iterations when $K=2$ (Fig. 5(a)) and $K=3$ (Fig. 5(b)). A direct comparison between Fig. 4(b) and Fig. 5(a), and between Fig. 4(d) and Fig. 5(b), reveals that the benefits of segmentation appear already from the first iterations, and can be hence exploited also when the number of IC cycles have to be kept low, as for example in situations where possible delay constraints must be satisfied.
A further observation regarding this second set of results concerns the substantially good agreement between theory and simulations. The moderate differences are due to three main reasons. The first one is the deviation, for $i \geq 2$, from the multinomial assumption used to evaluate the average capture probability in (36). In fact, as more segments are cancelled from the corresponding slices, the distribution of the remaining collided segments becomes farther from the multinomial one. The second reason is the lack of perfect independence among the $\alpha_{n, n^{\prime}}(\varrho ; \varsigma)$ values in (39). This implies that, actually, the probabilities of capturing $n^{\prime}$ over $n$ packets for $n^{\prime}=1, \ldots, n$ are partly correlated, and hence are not exactly binomially distributed. The third reason concerns the actual fading statistic for $i>1$. As $i$ increases, in fact, the powers of the remaining segments are no more completely representative of an exponentially distributed population, since they correspond just to the even more degraded ones. Observe that this problem can arise for any fading statistic, since the removal of the some segments unavoidably changes the statistic of the remaining 


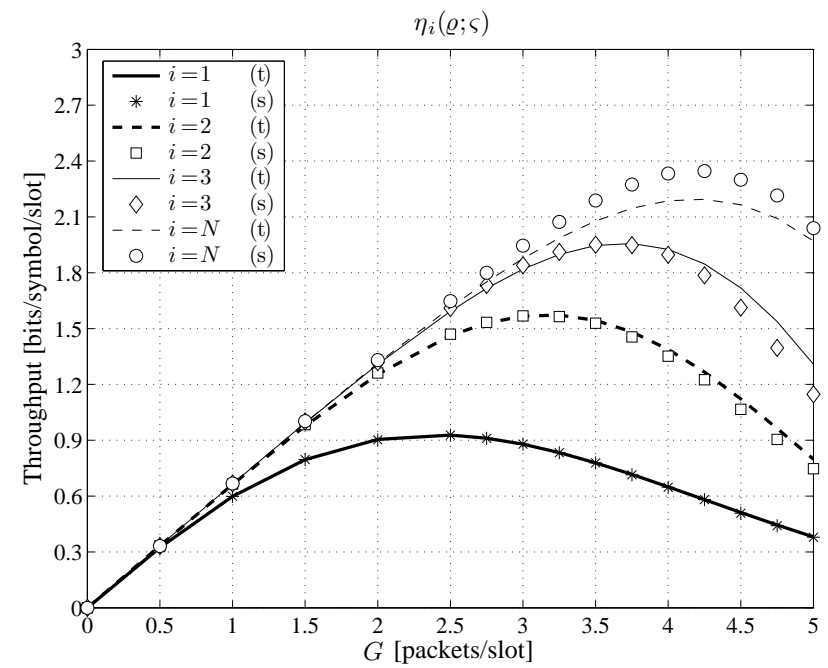

(a)

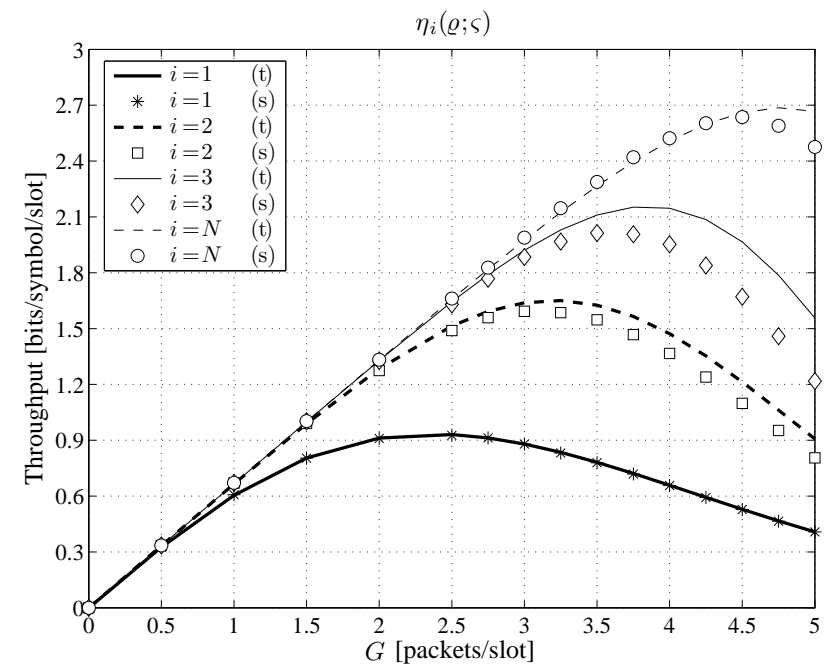

(b)

Fig. 5. Throughput as a function of the offered load at different iterations for $L=3$ in a fast fading scenario (t: theory, s: simulation): (a) $K=2$, (b) $K=3$.

ones. This issue has been also outlined in [26], by showing that the density evolution analysis originally adopted in [9] may become less accurate in the presence of capture, even when it involves just the single segment and erasure packet decoding is assumed, because of the interdependence among slices and the approximate SINR modeling after each IC cycle. In the here proposed model, the difficulty in theoretically modeling the powers of the remaining segments is further enhanced by the fact that the capture event, according to (11), jointly accounts for all the segments of the packet.

Bearing in mind all these three discussed aspects, it is interesting to observe that the adoption of the same capture and fading model at each iteration is slightly optimistic, while the effects of the binomial approximation are more difficult to predict, since they may lead to an overestimation or to an underestimation of the performance, depending on the specific scenario. This explains why, with respect to the Monte carlo simulations, the theory, which does not aim to provide a throughput bound (upper or lower), but a throughput approximation, is better in some situations, and worse in others. Even with these differences, however, the matching between theory and simulation may be considered satisfactory, also considering the acceptable level of complexity of the analysis. Regarding this latter issue, it is worth to remark that, to the best authors' knowledge, the currently existing theories for FSA with IC providing the performance as a function of the iteration are two: the throughput upper bound for the CRDSA scheme in the absence of capture and segmentation [7], and the packet erasure probability for the IRSA scheme in the presence of given (uncorrelated) capture coefficients and in the absence of segmentation [8]. By consequence, the here developed analysis represents the first attempt of theoretically modeling the performance of an FSA system with IC, capture, and segmentation by deriving the capture probability in nonideal channel conditions and by allowing the evaluation of the throughput at each iteration.

\section{Practical system}

This third subsection aims to check the applicability of the formulated decoding criterion to a practical scenario in which an actual encoder, operating on real sequences of bits, is employed. To this aim, a QPSK modulation (order $\xi=4$ ) and a robust turbo encoder of rate $\rho=1 / 3$, implemented in c++ language, are adopted to generate the selected rate $\varrho=\rho \log _{2} \xi=$ $2 / 3$. In particular, the turbo encoder, whose characteristics are specified in [27], is a parallel concatenated convolutional code with two constituent encoders and an internal interleaver. The uncoded packet has a length of 1340 bits, while the coded one has a length of $1340 / \rho+12$ bits. A scrambler manipulates the coded bits to improve the symmetry of the code before the segmentation. At the destination, the received bits of each segment are manipulated by a descrambler and the received sequence is decoded by an iterative turbo decoding algorithm. Since an actual modulation is now employed, the usage of the Shannon bound in (7) to model the relationship between the equivalent rate and the SINR for the generic segment of a packet might result too optimistic. To overcome this limitation, we adopt a more suitable relationship, which has been specifically derived for binary and quadrature PSK modulations in [28]. According to this formulation, the rate $\Lambda_{l}$ achievable in the presence of a SINR $X_{l}$ when a QPSK modulation is used is approximated, for $l=1, \ldots, L$, by [28]:

$$
\Lambda_{l} \cong 2\left\{1-\exp \left[a_{1}\left(\frac{X_{l}}{2 b}\right)^{a_{2}}+a_{3}\right]\right\},
$$

where $a_{1} \cong-1.286, a_{2} \cong 0.9308, a_{3} \cong 0.010$ are proper coefficients, and $b$ is a margin introduced to account for the finite length of the codeword, the non-ideality of the code, and its non-perfect symmetry. For the adopted code, a margin $b=$ $2.5 \mathrm{~dB}$ has been selected. With respect to the exact definition involving the $Q$-function, (46) has the advantage of being invertible simultaneously maintaining an approximation error lower than $1 \%$ for SINR values not too close to zero. For this 


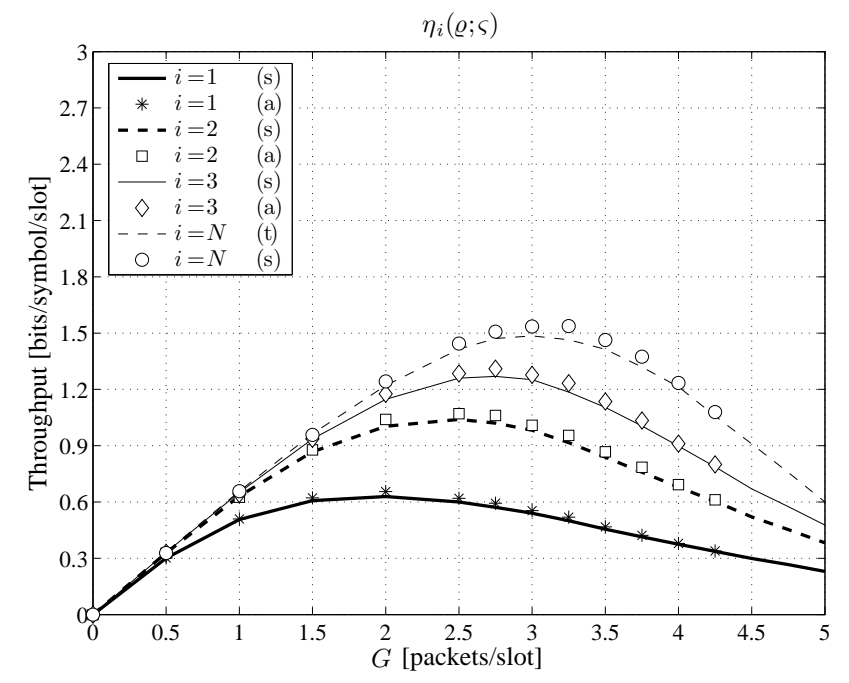

(a)

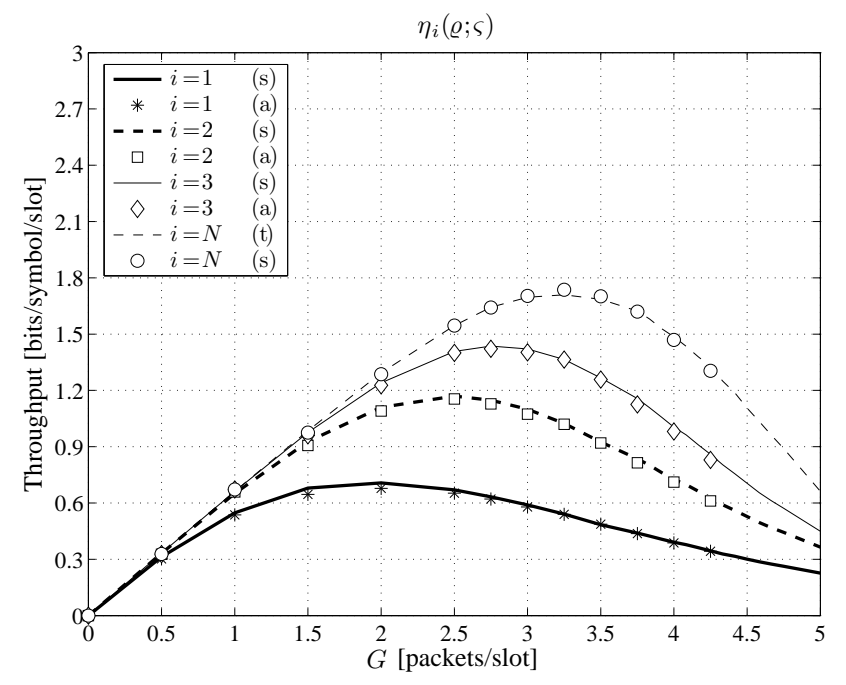

(b)

Fig. 6. Comparison with a practical system: throughput as a function of the offered load at different iterations for $K=3$ in a slow fading scenario (s: simulation, a: actual performance): (a) $L=2$, (b) $L=3$.

reason, it is used in (11) to evaluate the equivalent rate of each transmission carried out using the adopted code-modulation pair. The subsequent throughput evaluation for the FSA system is carried out numerically, since, even if the cdf of $\Lambda_{l}$ may still be analytically derived, a possible equivalent rate analysis does not provide closed-form expressions. The actual performance of the system is hence obtained by averaging the results corresponding to $Q^{\prime}$ realizations, in which, differently from the pure Monte Carlo simulations, coded and QPSK-modulated sequences are actually present in each slice. Besides, in this case, the number of realizations $Q^{\prime}$ is not a priori specified, since the stopping criterion is based on the variance of the throughput. In particular, the realizations are repeated until the confidence interval of the throughput (not reported in the figures to maintain their readability) becomes negligible.

The first set of results is presented in Fig. 6, which reports the throughput as a function of the offered load at the different iterations for $K=3$ in a slow fading scenario considering $L=2$ (Fig. 6(a)) and $L=3$ (Fig. 6(b)) segments. In this subsection, the simulated curves (using the equivalent rate) are identified by lines, while those derived from the implementation of the actual encoder (not using the equivalent rate) are identified by markers. From this figure we can outline two main observations. Firstly, as expected, adopting a practical encoder and a real modulation, the throughput decreases with respect to that obtained using the Shannon bound. However, even in this situation, the formulated decoding criterion based on the equivalent rate remains reliable in predicting the system performance, as it results from the very good agreement between the simulated curves and the actual ones. This reveals a significant advantage of the technique here presented for modeling the code behavior, since the CPU time required to obtain a curve is in the order of one day for the actual performance, which relies on the real encoder, and in the order of just few minutes for the simulation, which relies on the adopted capture criterion based on the equivalent rate.

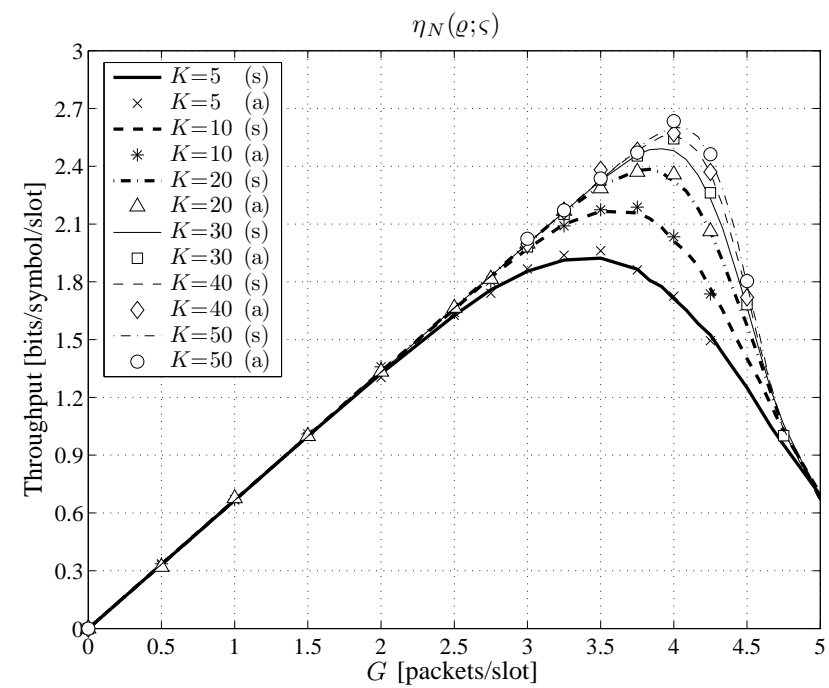

Fig. 7. Comparison with a practical system: throughput as a function of the offered load for $L=3$ and different number of slots in a slow fading scenario ( $\mathrm{s}$ : simulation, a: actual performance).

The last set of results of this section shows the throughput as a function of the offered load in a slow fading scenario for $L=3$ considering also RAFs with tens of slots (Fig. 7). Remembering, as specified at the end of Subsection V-A, that a normalized population size $\sigma=N / K=5$ users per slot is adopted, one may notice that the increase of the number of slots corresponds also to the increase of the number of contending users. The figure confirms that a larger RAF allows the system to sustain a higher load and to achieve a higher throughput. However, the performance improvement becomes less significant as $K$ gets higher, thus revealing a sort of saturation when longer RAFs are adopted. Furthermore, we may observe that the throughput closely approaches the straight line $\eta_{N} \cong \varrho G$ when the offered load is low, thus, 


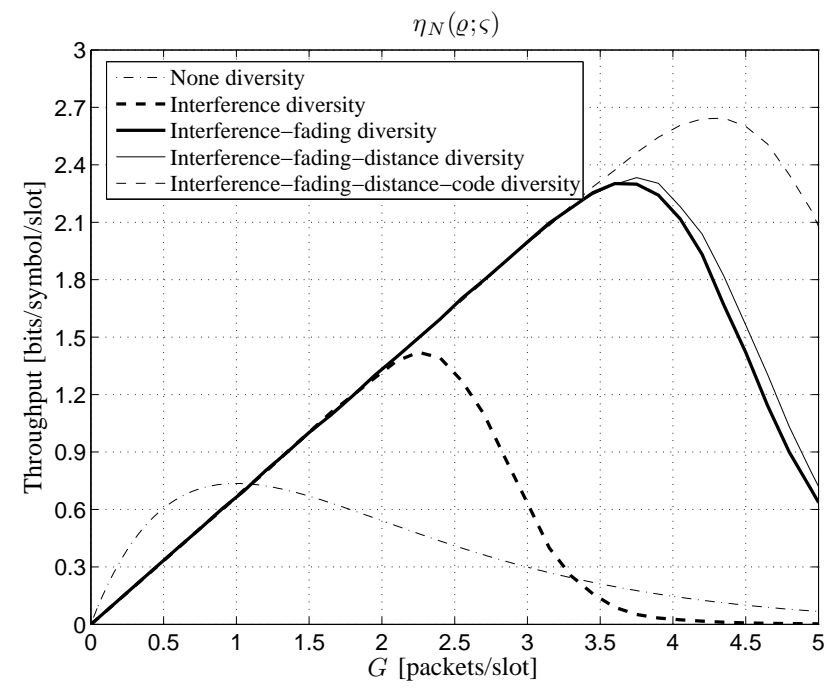

Fig. 8. Impact of different kinds of diversity on the throughput.

in moderate traffic conditions, all the information bits of the packets are successfully received. As the RAF gets longer, this closeness is maintained for higher $G$ values, even if a more significant performance reduction occurs once the maximum throughput is reached. This behavior is consistent with the results in [9, Fig. 7] obtained for the CSA scheme, which reveal that, as the RAF duration increases, the actual throughput better fits the asymptotic one. Of course, this consistency concerns the sole shape of the curves, since, in [9], the maximum throughput and the corresponding offered load are both lower than one, while, in the here investigated scenario, they may become higher than one thanks to the capture effect.

\section{IMPLEMENTATION ISSUES}

This section finally discusses the possible implementation of a segmented FSA system in a real network. All curves are obtained for $N=300, K=20, \varsigma=-30 \mathrm{~dB}$, and $\varrho=2 / 3$.

The first discussed issue concerns the presence of a general environment in which the users adopt different codes and are located at different distances from the common receiver. To investigate this situation, Fig. 8 shows the throughput achievable by increasing the level of diversity. As reference consider the absence of any diversity, that is, the common FSA with throughput $2 G \exp (-G)$ (the factor two is due to the adoption of a QPSK modulation), which remains the preferable solution for low channel loads. For higher loads, a first improvement is provided by interference diversity, which derives from the introduction of segmentation, while a second improvement by the presence of fading, which provides a form of power diversity. A third moderate throughput increase for higher channel loads can occur when the transmitters, rather than being at the same distance from the receiver (i.e., $\zeta=0 \mathrm{~dB}$ identical for all sources), are instead uniformly distributed over an annulus centered at the receiver with inner radius of one meter and outer radius of ten meters, in a propagation environment characterized by a path-loss exponent $\epsilon=3$. This leads to different transmitter-receiver distances, which,

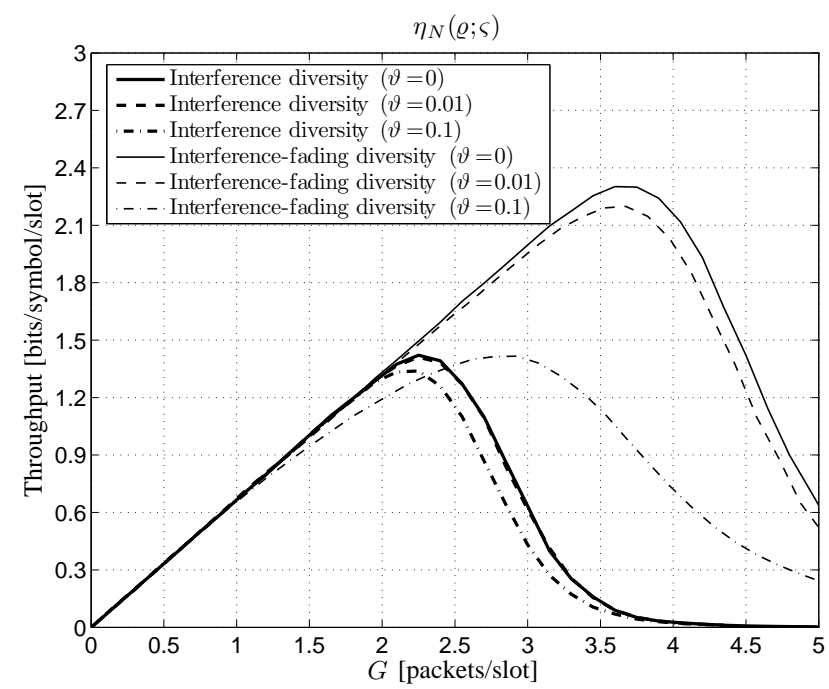

Fig. 9. Impact of imperfect IC on the throughput.

in turn, represents a further form of power diversity. To make more complete this view, code diversity is also added. This kind of diversity derives from the adoption of different code rates with different probabilities. In particular, with reference to the optimized codes in [9], the here considered case refers to a scheme in which an uncoded packet is split in two data segments and an access scheme rate $1 / 3$ is selected. This leads to the possible numbers of encoded segments $L=3,4,5,11,12$ generated with probabilities $p_{3}=0.259929, p_{4}=0.053247$, $p_{5}=0.447058, p_{11}=0.105258, p_{12}=0.134509$ [Table I, 9]. Note that, for coherence, the access scheme rate $1 / 3$ in [9] has been chosen equal to the rate $\rho=1 / 3$ so far adopted for the channel encoder. Thus, since each uncoded packet is split in two data segments, $L=2 \rho=6$ encoded segments are used for all cases in which interference diversity is present and code diversity is absent. Regarding Fig. 8, one may notice from a direct comparison between the cases corresponding to interference-fading diversity and interference-fading-distance diversity, that, in the assumed path-loss scenario, the main contribution to the power diversity effect is provided by the fading phenomenon.

The second discussed implementation issue concerns the performance achievable in the presence of channel estimation errors, which may arise in practical systems. The inaccuracy in reconstructing the waveforms corresponding to the decoded segments leads in fact to imperfect IC, which leaves a residual interference power in the involved slices. This residual is usually assumed proportional to the power of the segment before cancellation through a coefficient $\vartheta$, which is selected between 0 and 1 [29], [30]. Following this approach, Fig. 9 shows the throughput for the interference diversity and interference-fading diversity cases when $1 \%$ and $10 \%$ residuals are considered. The most interesting aspect put into evidence by this figure is the considerably different impact of the interference residual in the absence and in the presence of fading. In particular, the interference diversity scenario results much more robust against imperfect IC as compared 


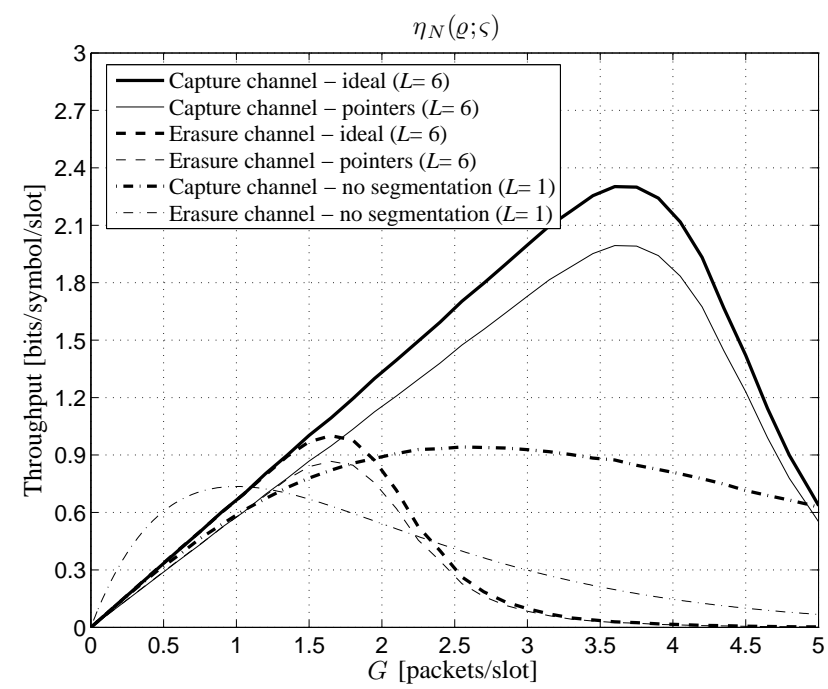

Fig. 10. Impact of channel model and pointers on the throughput.

to the interference-fading diversity one, thus revealing that the diversity intentionally introduced at the system level through packet segmentation substantially maintains its benefits, while the diversity provided by the propagation environment is very sensitive to the accuracy of the channel estimation process. This second behavior is mainly due to the high power realizations of the randomly generated powers, which, when not perfectly cancelled, leave significant interference residuals that make difficult the decoding of the remaining segments.

A third relevant issue for the actual implementation of a segmented FSA system concerns the problem, for the destination, of finding the $L$ slices where the $L$ segments of a given packet lie. This problem can be solved by including, in each encoded segment of a packet, a header containing the pointers to the slices of the RAF in which the segments of that packet have been placed [9]. The header is transmitted at the same rate $\varrho=2 / 3$ used for the information, that is using a QPSK modulation and a code rate $\rho=1 / 3$. In this way, the capture of just one segment may enable the destination to acquire the position in the RAF of all the other segments of the same packet. Regarding the possibility of decoding the pointers of at least one segment, one may formulate the following observation. Given the adopted decoding requirement $R=$ $\left(\sum_{l=1}^{L} \lambda_{l}\right) / L \geq \varrho$, we necessarily have, in case of successful packet decoding, that the highest rate $R_{\max }=\max \left\{\lambda_{1}, \ldots, \lambda_{L}\right\}$ is surely larger or equal to $R$ and hence larger or equal to $\varrho$. Thus, if each header is encoded by the same encoder used for the full packet and if that packet is decodable (i.e., captured), the header of the best segment of that packet is also decodable, so that the pointers become available and the decoding IC process can start. Concerning this latter aspect, it is worth to remark that the condition of decoding at least one segment (i.e., $R_{\max } \geq \varrho$ ), is much less stringent than that of decoding the entire packet (i.e., $R \geq \varrho$ ), since the relationship $R_{\max } \geq R$ always holds. This implies that, if $R_{\max }<\varrho$, no pointers are available, but in this situation they would have been useless, since the condition $R \geq \varrho$ would not have been satisfied anyway. Otherwise, if $R_{\max } \geq \varrho$, the pointers are available, while the condition $R \geq \varrho$ can be satisfied or not (i.e., packet capture can occur or not). Therefore, the prerequisite of pointers' availability before the beginning of the decoding process of the entire packet, which is requested by a real system, is verified.

Fig. 10 shows the impact of the pointers' overhead on the throughput when, as in the previous section, a payload of 1340 bits is adopted. The figure, which also reports the performance obtained adopting the erasure channel, is obtained considering that each segment must contains $H=(L-$ 1) $\log _{2}(K L)$ bits to identify the position of the other $L-1$ segments in the RAF, thus the overall overhead due to the pointers is $L H$. One may notice that, regardless of the specific channel model, the benefits of segmentation for higher channel loads remain relevant also when this overhead is included. In particular, the erasure channel necessarily provides a lower performance, since, for packet decoding, it requires the reception of at least a certain number of clean (or cleaned after IC) segments, a strong constraint that is not essential in more realistic capture scenarios. Besides, as outlined in [9], the overhead $L H$, which can be more pronounced for small packets, can be reduced by suitable techniques based on code indexes and pseudo-random seeds.

Fig. 10 is also useful to respond to a basic question concerning the introduction of novel functionalities in a wellestablished FSA system: is the complexity increase due to the implementation of segmentation justified by the obtained performance improvement, or it results substantially hidden by the already present capture effect? A direct comparison between the curves derived in the presence of capture for $L=1$ (thick dash-dotted), and for $L=6$ accounting for the overhead due to the pointers (thin solid) provides the answer. For very low $G$ values, the throughput without segmentation is slightly higher because of the absence of the pointers (useless when segmentation is not adopted). In almost the rest of the $G$ range, instead, the use of segmentation provides a significant performance increase. This allows one to conclude that the throughput benefits seem to fully justify the increased complexity necessary to enable segmentation in FSA systems.

\section{CONCLUSIONS}

The impact of capture, packet segmentation, and slot slicing on the performance of an FSA system using IC has been theoretically investigated through the elaboration of a throughput analysis covering slow and fast fading conditions. This analysis has been developed using a suitable reception criterion based on the selected code rate and on the equivalent one associated to the received segments belonging to a packet. All theoretical results have been validated by Monte Carlo simulations, which have been also employed to check the applicability of the reception criterion to a practical system involving a QPSK modulation combined with a turbo encoder.

The most significant theoretical advance has been the derivation of a completely analytical framework, providing the throughput of an FSA system for each capture/IC iteration in the presence of segmentation and slicing. Both theory 
and simulations have clearly shown the benefits of these two mechanisms, which, producing an interference diversity among the different slices, provide an increase of the capture probability together with a good robustness against imperfect IC. The validation of the practical system has revealed that the concept of equivalent rate might be viewed as a useful element to insert not only in theoretical tools, but also in network simulators, with the aim of improving their physical layer model, while maintaining a limited simulation time. From the implementation point of view, the insertion of suitable pointers in the header of each segment to allow the recovery of the position of the other segments of the packet, has proved to be feasible, since the resulting overhead has not significantly affected the throughput gain provided by segmentation. This suggests that next-generation communication standards adopting Aloha-based random access schemes might be usefully realized including segmentation as a possible functionality, which, triggering a beneficial interference diversity phenomenon, can be helpful for managing large amounts of contending traffics in forthcoming ultra-dense networks.

\section{REFERENCES}

[1] L. Kleinrock and F.A. Tobagi, "Packet switching in radio channels: Part I - Carrier sense multiple-access modes and their throughput-delay characteristics," IEEE Trans. Commun., vol. 23, no. 12, pp. 1400-1416, Dec. 1975.

[2] J.L. Massey, "Collision-resolution algorithms and random-access communications," in Multi-User Communication Systems, ser. 265, G. Longo, Ed. New York: Springer, 1981, pp. 73-137.

[3] K.A. Agha, P. Jacquet, and N. Vvedenskaya, "Analysis of the priority stack random-access protocol in W-CDMA systems," IEEE Trans. Veh. Technol., vol. 51, no. 3, pp. 588-596, May 2002.

[4] F. Babich, M. Comisso, A. Crismani, and A. Dorni, "On the design of MAC protocols for multi-packet communication in IEEE 802.11 heterogeneous networks using adaptive antenna arrays," IEEE Trans. Mobile Comput., vol. 14, no. 11, pp. 2332-2348, Nov. 2015.

[5] L.G. Roberts, "ALOHA packet systems with and without slots and capture," Telenet Commun. Corp., Washington, DC, USA, Tech. Rep. ARPANET System Note 8 (NIC11290), June 1972.

[6] G.L. Choudhury and S.S. Rappaport, "Diversity ALOHA - A random access scheme for satellite communications," IEEE Trans. Commun., vol. 31, no. 3, pp. 450-457, Mar. 1983.

[7] E. Casini, R. De Gaudenzi, and O. del Río Herrero, "Contention resolution diversity slotted ALOHA (CRDSA): An enhanced random access scheme for satellite access packet networks," IEEE Trans. Wireless Commun., vol. 6, no. 4, pp. 1408-1419, Apr. 2007.

[8] G. Liva, "Graph-based analysis and optimization of contention resolution diversity slotted ALOHA," IEEE Trans. Commun., vol. 59, no. 2, pp. 477-487, Feb. 2011.

[9] E. Paolini, G. Liva, and M. Chiani, "Coded slotted ALOHA: A graphbased method for uncoordinated multiple access," IEEE Trans. Inf. Theory, vol. 61, no. 12, pp. 6815-6832, Dec. 2015.

[10] Y. Liu, Z. Qin, M. Elkashlan, Y. Gao, and L. Hanzo, "Enhancing the physical layer security of non-orthogonal multiple access in large-scale networks," IEEE Trans. Wireless Commun., vol. 16, no. 3, pp. 16561672, Mar. 2017.

[11] J.-B. Seo, B.C. Jung, and H. Jin, "Nonorthogonal random access for 5G mobile communication systems," IEEE Trans. Veh. Technol., vol. 67, no. 38 , pp. 7867-7871, Aug. 2018.

[12] C. Namislo, "Analysis of mobile radio slotted ALOHA networks," IEEE Trans. Veh. Technol., vol. 33, no. 3, pp. 199-204, Aug. 1984.

[13] J.C. Arnbak and W. van Blitterswijk, "Capacity of Slotted ALOHA in Rayleigh-Fading Channels," IEEE J. Sel. Areas Commun., vol. 5, no. 2, pp. 261-269, Feb. 1987

[14] M. Zorzi, "Capture probabilities in random-access mobile communications in the presence of Rician fading," IEEE Trans. Veh. Technol., vol. 46, no. 1, pp. 96-101, Feb. 1997.
[15] L. Zhou, Y.-D. Yao, H. Heffes, and R. Zhang, "Investigation of slotted ALOHA under Nakagami fading with synchronized and asynchronous cochannel cells," IEEE Trans. Veh. Technol., vol. 52, no. 6, pp. $1642-$ 1651, Nov. 2003.

[16] Y. Li and L. Dai, "Maximum sum rate of slotted Aloha with capture," IEEE Trans. Commun., vol. 64, no. 2, pp. 690-705, Feb. 2016.

[17] E. Paolini, C. Stefanovic, G. Liva, and P. Popovski, "Coded random access: Applying codes on graphs to design random access protocols," IEEE Commun. Mag., vol. 53, no. 6, pp. 1408-1419, June 2015.

[18] F. Babich, "On the performance of efficient coding techniques over fading channels," IEEE Trans. Wireless Commun., vol. 3, no. 1, pp. 290-299, Jan. 2004.

[19] F. Babich and F. Vatta, "Robust, efficient and balanced (REB) ratecompatible puncturing schemes, for hybrid ARQ algorithms using turbo codes," in IEEE Int. Conf. Communications, Istanbul, Turkey, 2006, pp. 1154-1159.

[20] F. Babich and M. Comisso, "Coded slotted Aloha (CSA) with capture," in VDE Europ. Wireless, Catania, Italy, 2018, pp. 1-6.

[21] V. Krishnan, Probability and Random Processes. Ed. New York: Wiley, 2006

[22] M. Abramowitz and I.A. Stegun, Eds., Handbook of Mathematical Functions (Applied Mathematics Series 55). Washington, DC: NBS, 1964.

[23] R.D. Yates and D.J. Goodman, Probability and Stochastic Processes. Ed. New York: Wiley, 1999

[24] F. Babich and A. Crismani, "Cooperative coding schemes: Design and performance evaluation," IEEE Trans. Wireless Commun., vol. 11, no. 1, pp. 222-235, Jan. 2012.

[25] M. Franceschetti, M.D. Migliore, and P. Minero, "The capacity of wireless networks: Information-theoretic and physical limits," IEEE Trans. Inf. Theory, vol. 55, no. 8, pp. 3413-3424, Aug. 2009.

[26] E.E. Khaleghi, C. Adjih, A. Alloum, and P. Mühlethaler, "Near-far effect on coded slotted ALOHA," in IEEE Int. Symp. Personal, Indoor and Mobile Radio Communications, Montreal, QC, Canada, 2017, pp. 1-7.

[27] Universal Mobile Telecommunications System (UMTS); Multiplexing and channel coding (TDD), ETSI TS 125222 V3.1.1, 2000.

[28] F. Babich, A. Soranzo, and F. Vatta, "Useful mathematical tools for capacity approaching codes design," IEEE Commun. Lett., vol. 21, no. 9, pp. 1949-1952, Sep. 2017.

[29] S.P. Weber, X. Yang, J.G. Andrews, and G. de Veciana, "Transmission capacity of wireless ad hoc networks with outage constraints," IEEE Trans. Inf. Theory, vol. 51, no. 12, pp. 4091-4102, Dec. 2005.

[30] A. Zanella and M. Zorzi, "Theoretical analysis of the capture probability in wireless systems with multiple packet reception capabilities," IEEE Trans. Commun., vol. 60, no. 4, pp. 1058-1071, Apr. 2012.

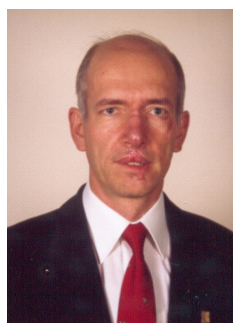

Fulvio Babich (SM'03) received the doctoral degree in electrical engineering, from the University of Trieste (Italy), in 1984. He worked for Telettra on optical communications and with Zeltron (Electrolux group) as Company Head in Home System European projects. Currently, he is an Associate Professor of digital communications at the Department of Engineering and Architecture of the University of Trieste. Fulvio Babich has served as co-chair at ICC 2005, 2011, 2014, and at WCSP 2012. He has been member of the Directive Board of CNIT (National Inter-University Consortium for Telecommunications, a non-profit Consortium among 37 Universities, whose main purpose is to coordinate and foster basic and applied research). His current research interests involve channel modeling, multiple access, channel encoding, error control, and cross-layer design.

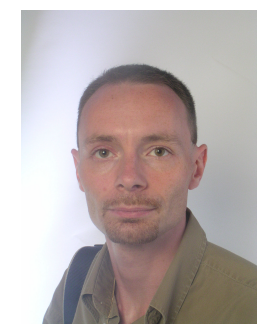

Massimiliano Comisso (M'09) received the Laurea and Ph.D. degrees in Information Engineering from the University of Trieste (Italy). He worked for Alcatel on DWDM systems and collaborated with Danieli Automation on NDE techniques. Currently, Massimiliano Comisso is an Assistant Professor at the Department of Engineering and Architecture of the University of Trieste. He is author/co-author of more than 60 international scientific papers. He has been Best Student Paper Award Finalist at GLOBECOM 2006 and received the Best Paper Award at CAMAD 2009. His research interests involve smart antenna systems, distributed wireless networks, millimeter-wave communications, antenna array synthesis, and small antennas. 